\title{
Smartphones in the secondary prevention of cardiovascular disease: a systematic review
}

\author{
Sandra J. Hamilton ${ }^{1 *}$, Belynda Mills ${ }^{1}$, Eleanor M. Birch ${ }^{1,2}$ and Sandra C. Thompson ${ }^{1}$
}

\begin{abstract}
Background: Cardiac Rehabilitation (CR) and secondary prevention are effective components of evidence-based management for cardiac patients, resulting in improved clinical and behavioural outcomes. Mobile health (mHealth) is a rapidly growing health delivery method that has the potential to enhance $C R$ and heart failure management. We undertook a systematic review to assess the evidence around mHealth interventions for $C R$ and heart failure management for service and patient outcomes, cost effectiveness with a view to how mHealth could be utilized for rural, remote and Indigenous cardiac patients.
\end{abstract}

Methods: A comprehensive search of databases using key terms was conducted for the years 2000 to August 2016 to identify randomised and non-randomised trials utilizing smartphone functionality and a model of care that included CR and heart failure management. Included studies were assessed for quality and risk of bias and data extraction was undertaken by two independent reviewers.

Results: Nine studies described a mix of mHealth interventions for CR (5 studies) and heart failure (4 studies) in the following categories: feasibility, utility and uptake studies; and randomised controlled trials. Studies showed that mHealth delivery for $C R$ and heart failure management is feasible with high rates of participant engagement, acceptance, usage, and adherence. Moreover, mHealth delivery of CR was as effective as traditional centre-based CR (TCR) with significant improvement in quality of life. Hospital utilization for heart failure patients showed inconsistent reductions. There was limited inclusion of rural participants.

Conclusion: Mobile health delivery has the potential to improve access to $C R$ and heart failure management for patients unable to attend TCR programs. Feasibility testing of culturally appropriate mHealth delivery for CR and heart failure management is required in rural and remote settings with subsequent implementation and evaluation into local health care services.

\section{Background}

Cardiovascular disease (CVD) is a leading cause of morbidity and mortality and the leading disease category for health-care expenditure in Australia [1, 2]. Cardiac Rehabilitation (CR) and secondary prevention are components of evidence-based management assisting patients with CVD (coronary artery disease, heart failure, atrial fibrillation and peripheral artery disease) return to an active and satisfying life through

\footnotetext{
*Correspondence: sandy.hamilton@uwa.edu.au

${ }^{1}$ Western Australian Centre for Rural Health, University of Western Australia,

35 Stirling Highway, Crawley, WA 6009, Australia

Full list of author information is available at the end of the article
}

improved clinical and behaviour outcomes and helps reduce the recurrence of cardiac events [3-5].

Cardiac rehabilitation (CR) is a coordinated multidimensional evidence-based strategy that aims to assist patients with CVD return to "an active and satisfying life and to prevent the recurrence of cardiac events" [6]. Secondary prevention, is defined as "healthcare designed to prevent recurrence of cardiovascular events or complications of CVD in patients diagnosed with CVD" [7]. Although these definitions are similar, CR may be time limited, whereas secondary prevention proposes a cardiac rehabilitation continuum where care is provided for the rest of a person's life according to need [7]. 
Cardiac rehabilitation is known to be underutilised: in Australia, attendance rates at traditional CR programs are estimated to be as low as $10-30 \%$ even in metropolitan areas, with even greater under-representation of rural, remote and Indigenous populations [5, 8]. Low CR attendance rates can reflect factors at the health service and broader system level, and well as health professional and patient related factors. These are significantly greater for people who live in rural and remote settings [8-11]. Systems and health professional related barriers limit accessibility through referral failure [8], absence of local CR programs and limited program places [8], program inflexibility $[8,10,11]$, and failure to meet the needs of individual patients [10].

Nearly one third of the Australian population reside in rural and remote areas, and despite similar rates of CVD, their cardiovascular outcomes are poorer than for those living in metropolitan areas [12]. Furthermore, the proportion of Aboriginal and Torres Strait Islander (hereafter Indigenous) Australians, known to have higher rates and earlier onset of CVD, increases with remoteness [13]. This vulnerable population is among those with more prevalent comorbidities who are less likely to receive, adhere to and complete $\mathrm{CR}[8,11]$, with its consequent suboptimal clinical benefit.

The care that patients receive is in part a function of the characteristics of health systems [14]. Inadequate health information systems and communication impede referral processes, service provision and continuity of care and contribute to referral failure, poor uptake and attendance and lower completion of $\mathrm{CR}$ for rural, remote and Indigenous patients [15]. For rural and remote patients, program availability and/or inflexibility, geographical location (distance, time and transport difficulties), hours of program scheduling, and cultural inappropriateness reduces accessibility and increases cost $[8,10,11]$. Alternative models of CR (Table 1), including patient-centred telehealth and community- or home-based CR, are preferred by many patients [5, 16-18]. These models encompass eight broad categories and have generally produced similar reductions in CVD risk factors compared with traditional outpatient CR [5].

Information and communication technologies (ICTs) have increasingly been incorporated into health care systems including innovative CR delivery $[19,20]$. ICTs include a variety of applications/ platforms which enable users to access, store, transmit and manipulate information electronically (eHealth). Advances have been enabled by the uptake of mobile technology, with 31 million mobile phone connections for a resident population of 23.6 million (131 mobile phones per 100 citizens in Australia) in June 2014 [21]. The uptake of smartphone technology in Australia has been rapid [22], with $89 \%$ of the 2014 Australian Mobile Phone Lifestyle Index survey
Table 1 Alternative models of cardiac rehabilitation

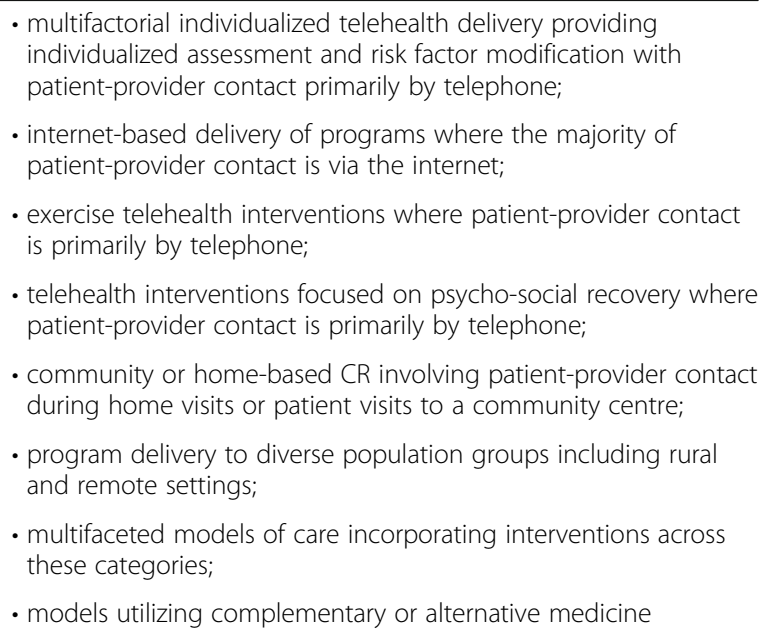

respondents aged $18-75$ years owning a smartphone. Mobile phone use was preferred (50\%) compared with a tablet device $(16 \%)$ or a personal computer (34\%). Health and wellbeing information had been accessed by $58 \%$ of the survey respondents within the last 12 months, and was used by $15 \%$ at least once weekly. Health and wellbeing applications (apps) were utilised by $27 \%$ of the survey respondents [22].

Mobile health (mHealth), a component of eHealth, is a rapidly growing health delivery methodology with the potential to impact on health care research, health care delivery and health outcomes [23]. Specifically, mHealth has the potential to enhance primary and secondary disease prevention and deliver interventions that are personalized, adaptive and sustainable, improve patient communication, access to health care services and treatment, and patient engagement and provide real-time medication monitoring and adherence support [23]. However, smartphone interventions may be limited by cost, especially for people with lower socioeconomic status who may have limited ability to pay the cost of receiving extra data via their smartphone. Furthermore, rural and remote populations may have poor access to data connectivity which limits smartphone use.

mHealth broadly refers to medical and public health practice supported by mobile devices [24] and includes mobile phones, smartphones, patient monitoring devices, personal digital assistants (PDAs) and other wireless devices. Mobile and smartphones provide the functionality of voice and short messaging service (SMS and/or text messaging), while smartphone functionality allows for downloaded programs (apps), numerous interfaces and specialized capabilities including third and fourth generation mobile telecommunications ( $3 \mathrm{G}$ and 4G systems), global positioning system (GPS) and Bluetooth connectivity $[24,25]$. mHealth enable 
consumers or providers to monitor health status through wireless diagnostic and clinical decision support [26]. The widespread adoption of smartphones and their integral role in people's lifestyle as a communication tool make them an attractive platform for the accurate capture of measurements and delivery of flexible health interventions or programs such as CR [20].

This systematic review examines smartphone interventions for comprehensive CR and heart failure rehabilitation/management for service and patient outcomes and how they can be utilised for cardiac patients in rural and remote settings.

\section{Methods}

\section{Search strategy}

A comprehensive search of electronic databases utilizing key terms relating to the research question was undertaken for the years 2000 to August 2016. The database search was supplemented by a manual search (pearling) of reference lists of included studies. Eligible studies were published in peer reviewed journals and in English. Randomised and non-randomised studies (randomised control trial (RCT)), quasi-experimental, or observational) with a prospective experimental study design (quantitative and qualitative) utilizing smartphone functionality and a model of care that included CR and/ or secondary cardiovascular prevention and heart failure rehabilitation were eligible for inclusion. Studies were excluded if they were retrospective, non-intervention studies, systematic reviews, study protocols, conference abstracts and papers reporting on content or technical development. We excluded studies which were primarily text messaging or web-based interventions.

The following databases were searched: PubMed, Medline, Academic Search Premier, CINAHL Plus, Embase, Google Scholar, and Cochrane Library. Boolean operators were utilized to combine key terms and $\mathrm{MeSH}$ terms including: rural, remote, regional, indigenous, cardiac rehabilitation, secondary cardiovascular prevention, healthcare applications, mHealth, eHealth, mobile, smartphone, computer, tele*, internet, web*, technolog*, communication, applications*, alternative methods, home-based (Additional file 1).

\section{Study selection}

Two reviewers independently screened potential articles for inclusion. The PRISMA guide (Preferred reporting items for systematic reviews and meta-analyses) was followed for study inclusion. Duplicate publications were removed, then titles and abstracts were screened for relevance. Full-texts of the remaining publications were retrieved by two reviewers and assessed against the inclusion and exclusion criteria (Table 2).

\section{Data extraction and analysis}

Studies initially considered suitable were reviewed by two independent reviewers using a template based on the Joanna Briggs Institute (JBI) Reviewers guidelines [27]. Publications were grouped by cardiac rehabilitation and heart failure and key information on study design, model of care, intervention and methods were synthesized.

Assessment of the level of evidence utilised the National Health and Medical Research Council's (NHMRC) Evidence Hierarchy [28]. Two reviewers also appraised the included studies for quality and risk of bias utilizing the Critical Appraisal Skills Programme (CASP) tools for methodological rigour of study design and the quality in reporting [29].

\section{Results}

The initial search identified 586 records, with an additional 21 records identified through a manual search of reference lists of included studies (pearling). Following removal of duplicates, abstract and full-title assessment, nine articles were considered eligible and included in the review (Fig. 1).

The included articles described a mix of mHealth interventions for CR (Tables 3, 4 and 5) and heart failure (Tables 6, 7 and 8) of two key types: (1) Feasibility, utility and uptake (FUU) studies: observational studies focussing on the feasibility and/or utility of

Table 2 Inclusion and exclusion criteria

P Patients eligible for Cardiac Rehabilitation and Secondary Prevention (acute coronary syndrome, re-vascularisation procedures, controlled heart fail-
ure and other vascular or heart disease [6,59]) or heart failure management.
I A model of care that utilized smartphone functionality (either app or Wireless Application Protocol (WAP) capabilities) for comprehensive
Cardiac Rehabilitation and Secondary Prevention or heart failure rehabilitation
C None, traditional cardiac rehabilitation or usual care
O Feasibility, utility, and uptake of mHealth; service outcomes (patient engagement, acceptance, adherence and completion, provider engagement
and acceptance, and cost effectiveness); patient outcomes (clinical, exercise capacity, knowledge, social and emotional, QOL); health service
utilisation.
E Retrospective studies; non-intervention studies; systematic reviews; study protocols; conference abstracts and non-cardiac rehabilitation or Key: $P$ Patient, I Intervention, C Comparison intervention, $O$ Outcomes, QOL Quality of life, E Exclusion criteria 


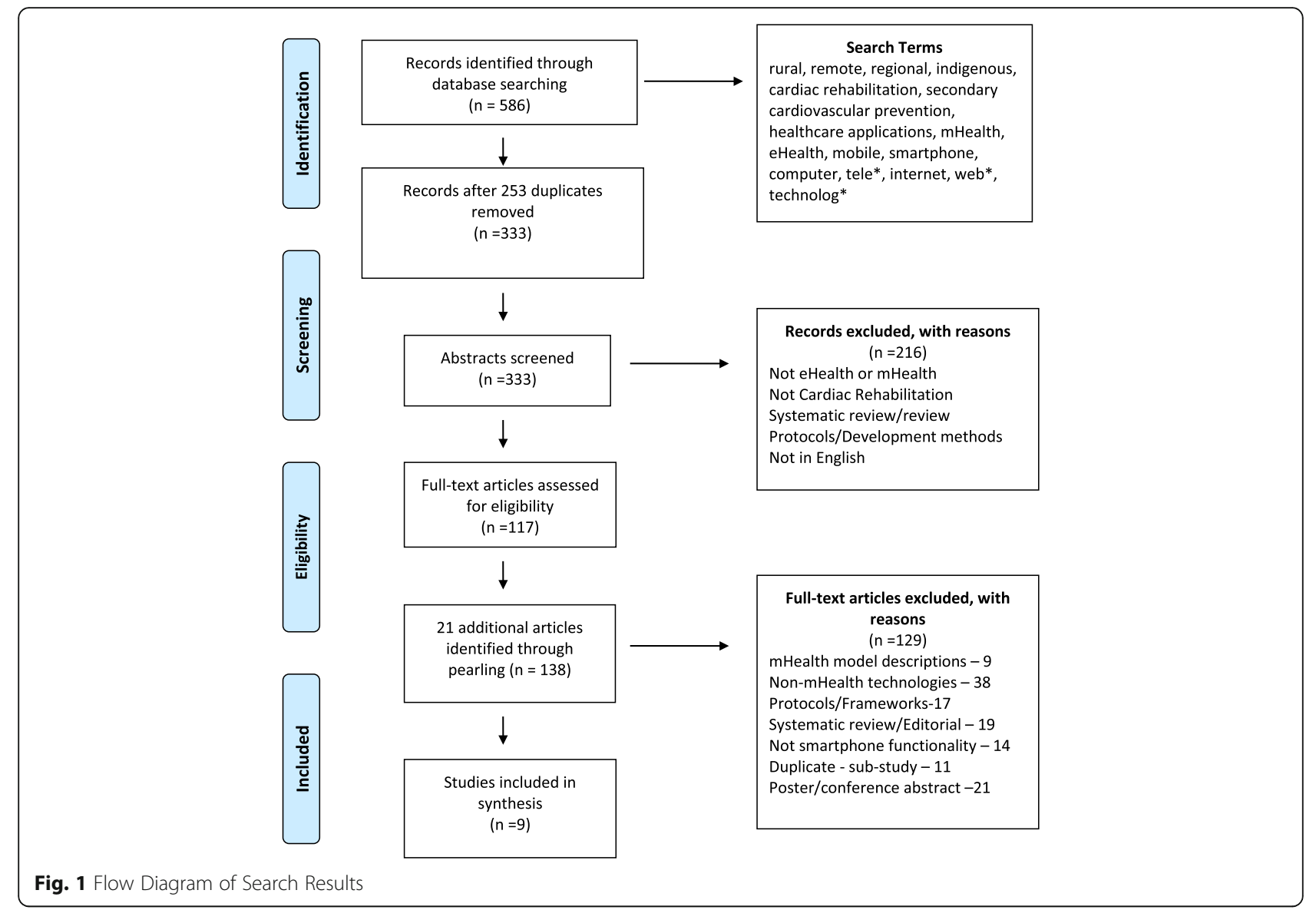

the intervention and reporting on participant uptake and acceptance; (2) RCTs: single-blind or open-label RCTs which compared a mHealth intervention with traditional CR (TCR) or usual heart failure management alone.

There was heterogeneity of the included studies related to study design, cardiac condition (ischaemic heart disease or heart failure) and outcome measures assessed. Levels of evidence, CASP score, theoretical framework and the differing outcome measures are reported in Tables 3 and 6 for CR and heart failure studies respectively. Outcomes are compared in Table 4 for CR and Table 7 for heart failure. CR and heart failure studies are summarised more fully in Tables 5 and 7 respectively.

\section{Cardiac rehabilitation}

Of the five articles focusing on CR, three were feasibility, utility and uptake studies and two were RCTs (Tables 3 and 4). Worringham et al. was the only study to report the inclusion of rural participants [30]. Varnfield and colleagues utilized a model of self-management combined with the core components of a comprehensive CR program [31, 32].
Feasibility, utility and uptake studies

A framework for the development and evaluation of mHealth has been developed by Whittaker and colleagues $[33,34]$. Steps in this process include pretesting, feasibility and pilot studies to test the content, regimen and processes of the intervention, and outcome assessment to assess technical feasibility, process issues and the acceptability of the intervention to participants and staff [33, 34].

The three investigations of feasibility, utility and uptake of mHealth CR utilised differing study designs (Table 4) [30, 31, 35], with all three studies based around smartphones programmed with additional applications for exercise and/or education delivery and remotely monitored patient data. Data was synchronised to a server via a secure web portal, giving program staff the ability to assess participant outcomes and provide feedback in real-time [30, 31, 35]. Technology and patientrelated measures are shown in Table 2 [30, 31, 35].

\section{Feasibility}

Delivering the core components of CR, either exercise alone or exercise and education via smartphone, was demonstrated to be technically feasible [30, 31, 35]. 
Table 3 Levels of evidence and outcome measures in cardiac rehabilitation studies

\begin{tabular}{|c|c|c|c|c|c|}
\hline & Worringham, 2011 & Forman, 2014 & Varnfield, 2011 & Blasco, 2012 & Varnfield, 2014 \\
\hline Country of Origin & Australia & USA & Australia & Spain & Australia \\
\hline \multicolumn{6}{|l|}{ Levels of evidence } \\
\hline Design & FUU & FUU & FUU & $\mathrm{RCT}$ & $\mathrm{RCT}$ \\
\hline Level of Evidence* & IV & IV & IV & $\|$ & $\|$ \\
\hline CASP score & $\mathrm{n} / \mathrm{a}$ & $\mathrm{n} / \mathrm{a}$ & $\mathrm{n} / \mathrm{a}$ & 7 & 8 \\
\hline Theoretical framework & & & $\checkmark$ & & $\checkmark$ \\
\hline \multicolumn{6}{|l|}{ Outcome measures } \\
\hline \multicolumn{6}{|l|}{ Technology } \\
\hline Feasibility & $\checkmark$ & $\checkmark$ & $\checkmark$ & $\checkmark$ & \\
\hline Usability & $\checkmark$ & $\checkmark$ & $\checkmark$ & & \\
\hline Technical problems & $\checkmark$ & & $\checkmark$ & & $\checkmark$ \\
\hline Acceptability & & $\checkmark$ & $\checkmark$ & $\checkmark$ & $\checkmark$ \\
\hline Engagement & $\checkmark$ & $\checkmark$ & & & \\
\hline Adherence & $\checkmark$ & $\checkmark$ & $\checkmark$ & $\checkmark$ & $\checkmark$ \\
\hline Usage & & & $\checkmark$ & & \\
\hline Task completion & & $\checkmark$ & & & \\
\hline \multicolumn{6}{|l|}{ Patient } \\
\hline Uptake & $\checkmark$ & & $\checkmark$ & & $\checkmark$ \\
\hline PROs & & $\checkmark$ & & & \\
\hline Program completion & $\checkmark$ & & & & $\checkmark$ \\
\hline Qualitative feedback & $\checkmark$ & $\checkmark$ & $\checkmark$ & & \\
\hline CV risk improvement & & & & $\checkmark$ & \\
\hline Physical activity & & & $\checkmark$ & $\checkmark$ & $\checkmark$ \\
\hline Step counter & & & $\checkmark$ & & $\checkmark$ \\
\hline 6MWT & $\checkmark$ & & & & $\checkmark$ \\
\hline Nutrition & & & $\checkmark$ & $\checkmark$ & $\checkmark$ \\
\hline Smoking status & & & & $\checkmark$ & \\
\hline Psychological distress & & & & & $\checkmark$ \\
\hline Depression & $\checkmark$ & & & & $\checkmark$ \\
\hline Anxiety & & & & $\checkmark$ & $\checkmark$ \\
\hline $\mathrm{QOL}$ & $\checkmark$ & & & $\checkmark$ & $\checkmark$ \\
\hline \multicolumn{6}{|l|}{ Self-efficacy } \\
\hline $\mathrm{BP}$ and $\mathrm{HR}$ & & & $\checkmark$ & $\checkmark$ & $\checkmark$ \\
\hline Weight & & & $\checkmark$ & & $\checkmark$ \\
\hline BMI & & & & $\checkmark$ & $\checkmark$ \\
\hline Waist circumference & & & & & $\checkmark$ \\
\hline $\mathrm{HbAl}_{\mathrm{c}}$ & & & & $\checkmark$ & $\checkmark$ \\
\hline Plasma lipid level & & & & $\checkmark$ & $\checkmark$ \\
\hline \multicolumn{6}{|l|}{$\mathrm{PVO}_{2}$} \\
\hline \multicolumn{6}{|l|}{ Medication adherence } \\
\hline Economic evaluation & & & & & $\checkmark$ \\
\hline
\end{tabular}

Key: $\checkmark$ Outcome measured, FUU Feasibility, Utility and Uptake study, RCT Randomised Control Trial, $n / a$ Not applicable, CASP Critical Appraisal Skills Programme, PROs Patient reported outcomes, CV Cardiovascular, $6 M W T$ Six minute walk test, QOL Quality of life, BP Blood pressure, HR Heart rate, BMI Body mass index, HbA1 $c$ Haemoglobin $\mathrm{Al}_{{ }_{1}} \mathrm{PVO}_{2}$ Peak oxygen uptake. *National Health and Medical Research Council's (NHMRC) Evidence Hierarch [28]. 
Hamilton et al. BMC Cardiovascular Disorders (2018) 18:25

Page 6 of 23

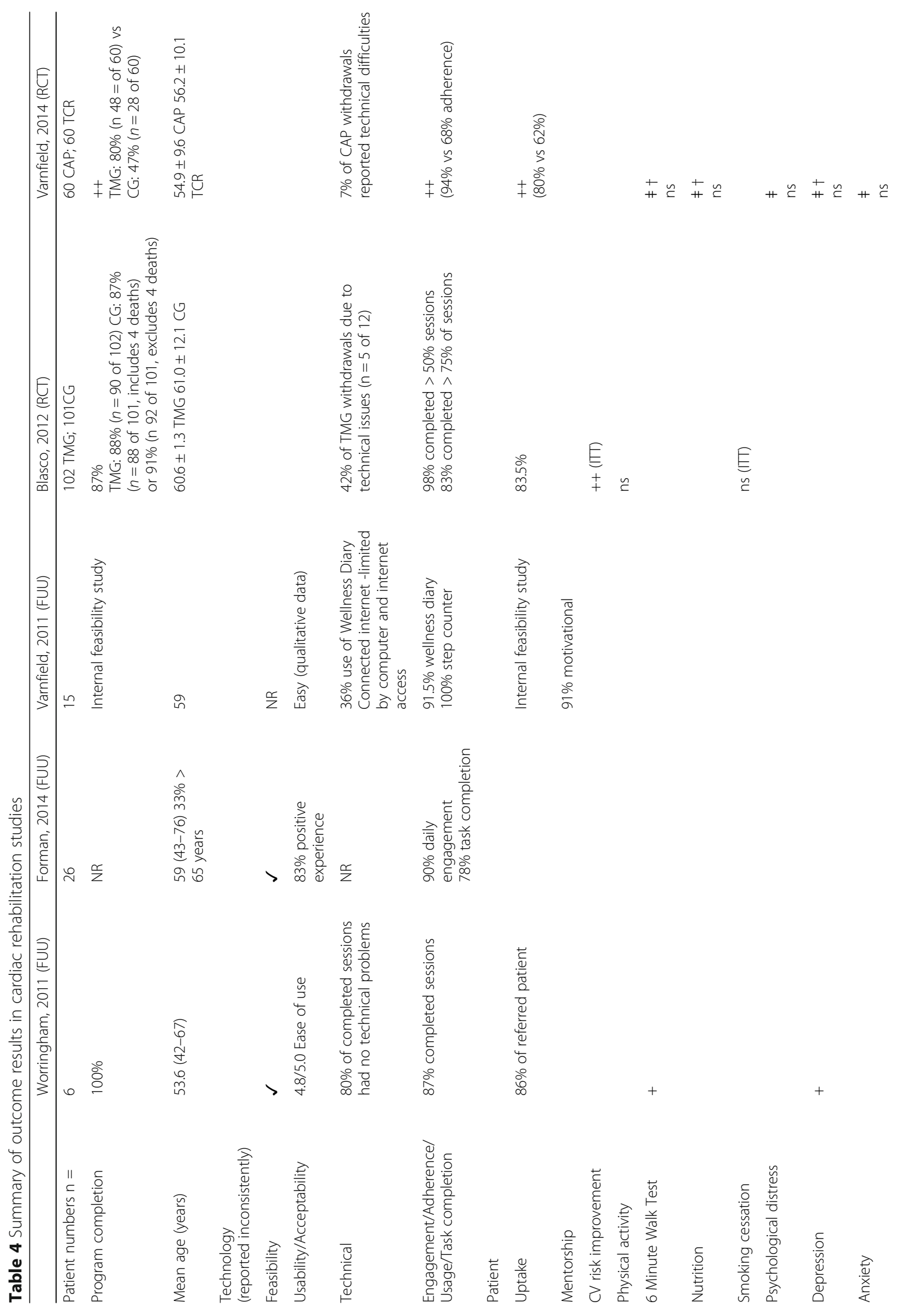


Hamilton et al. BMC Cardiovascular Disorders (2018) 18:25

Page 7 of 23

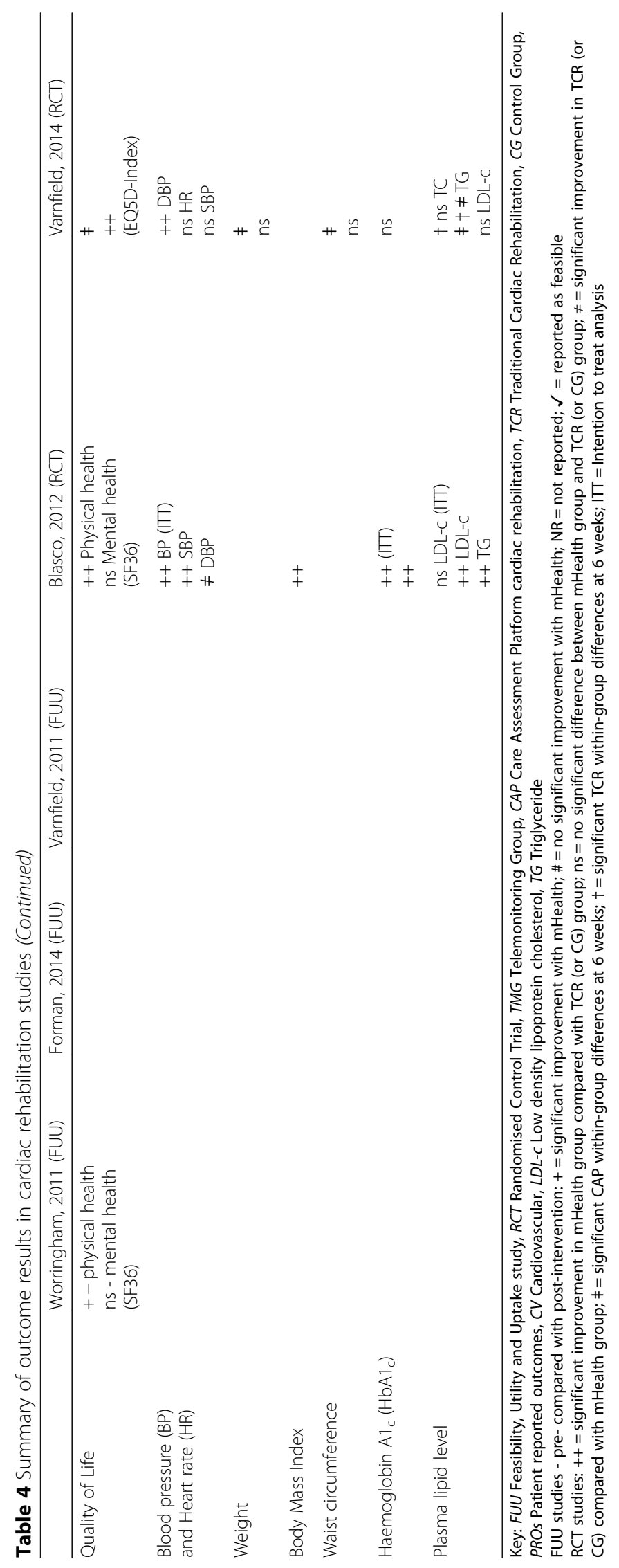




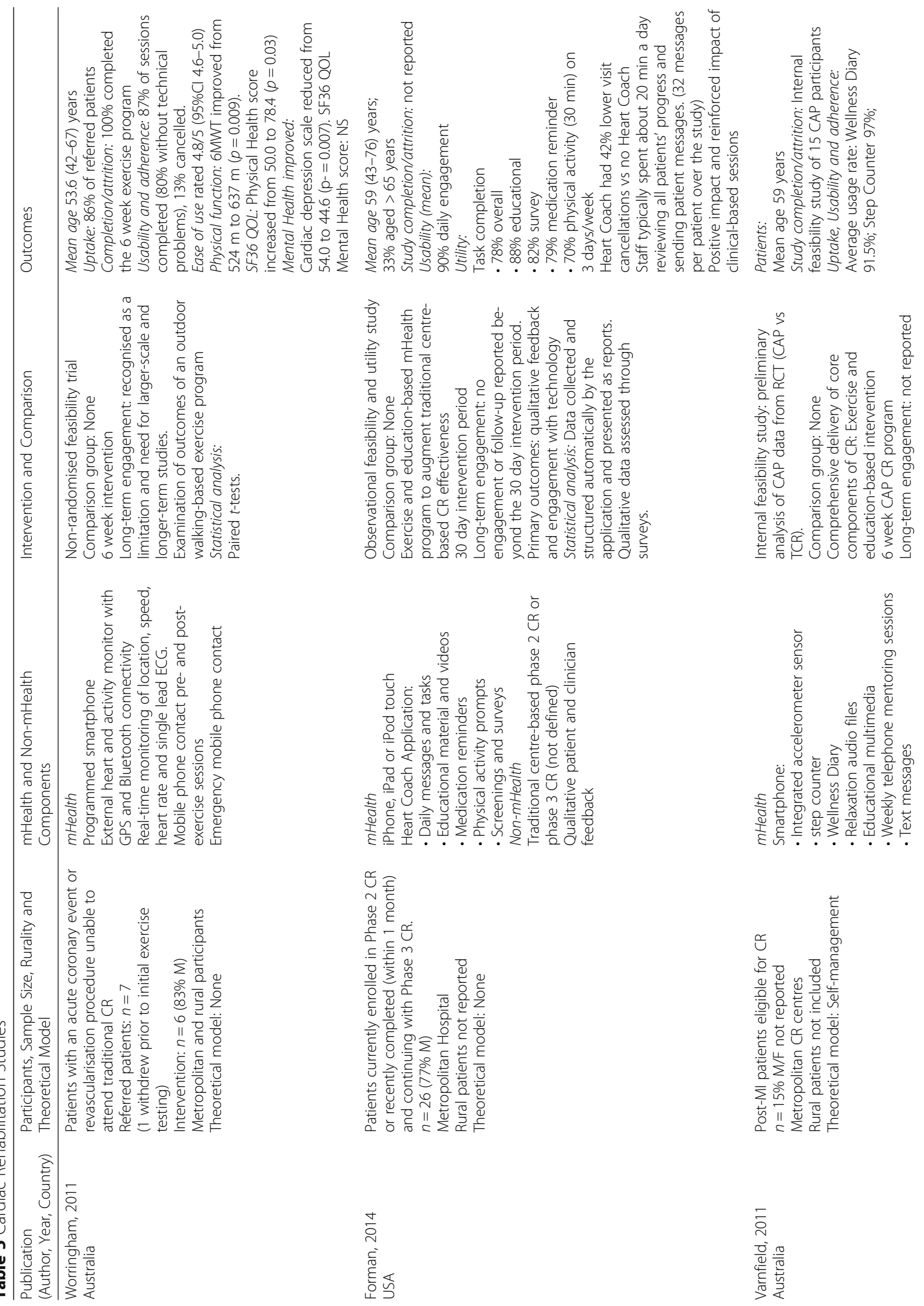




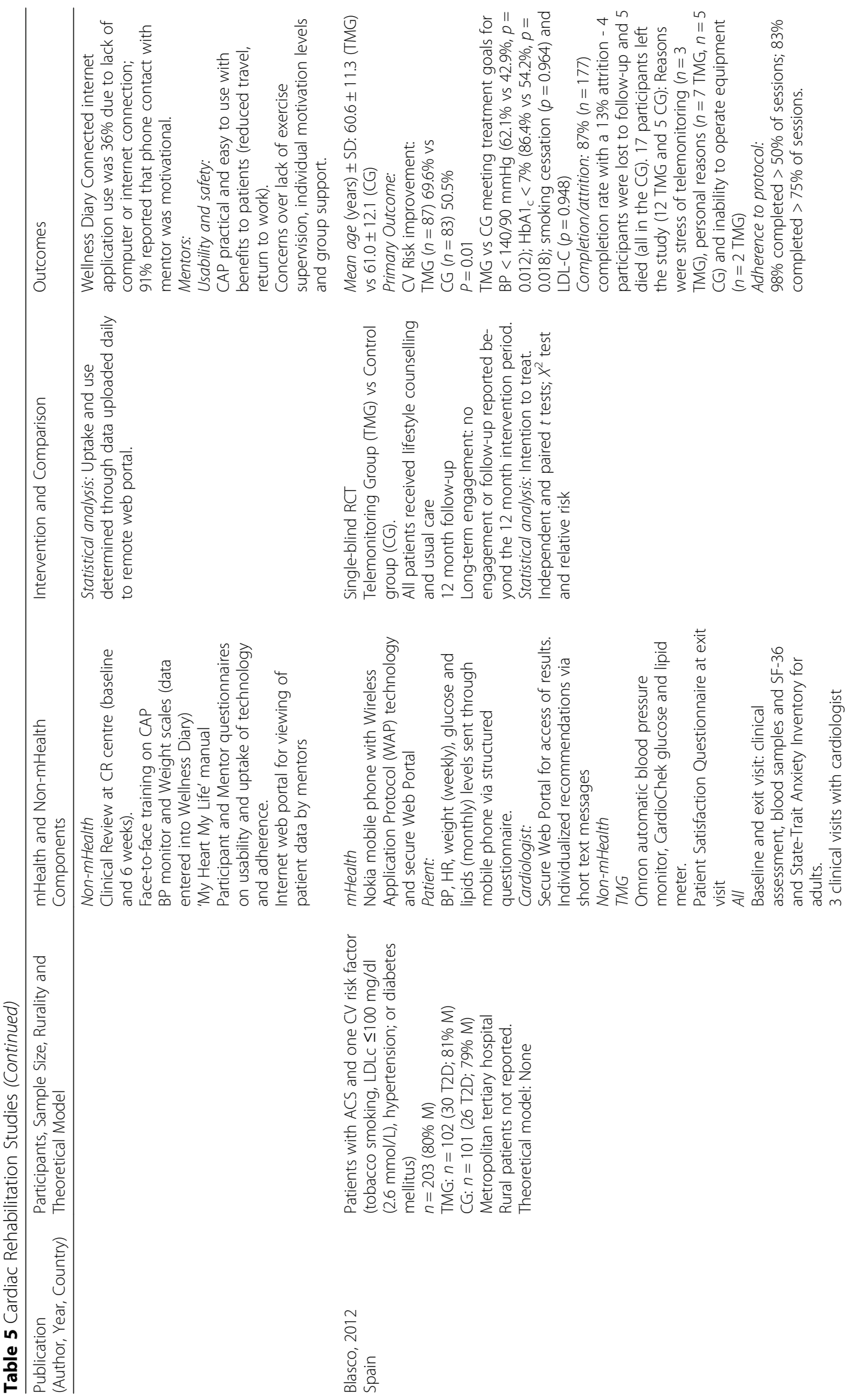




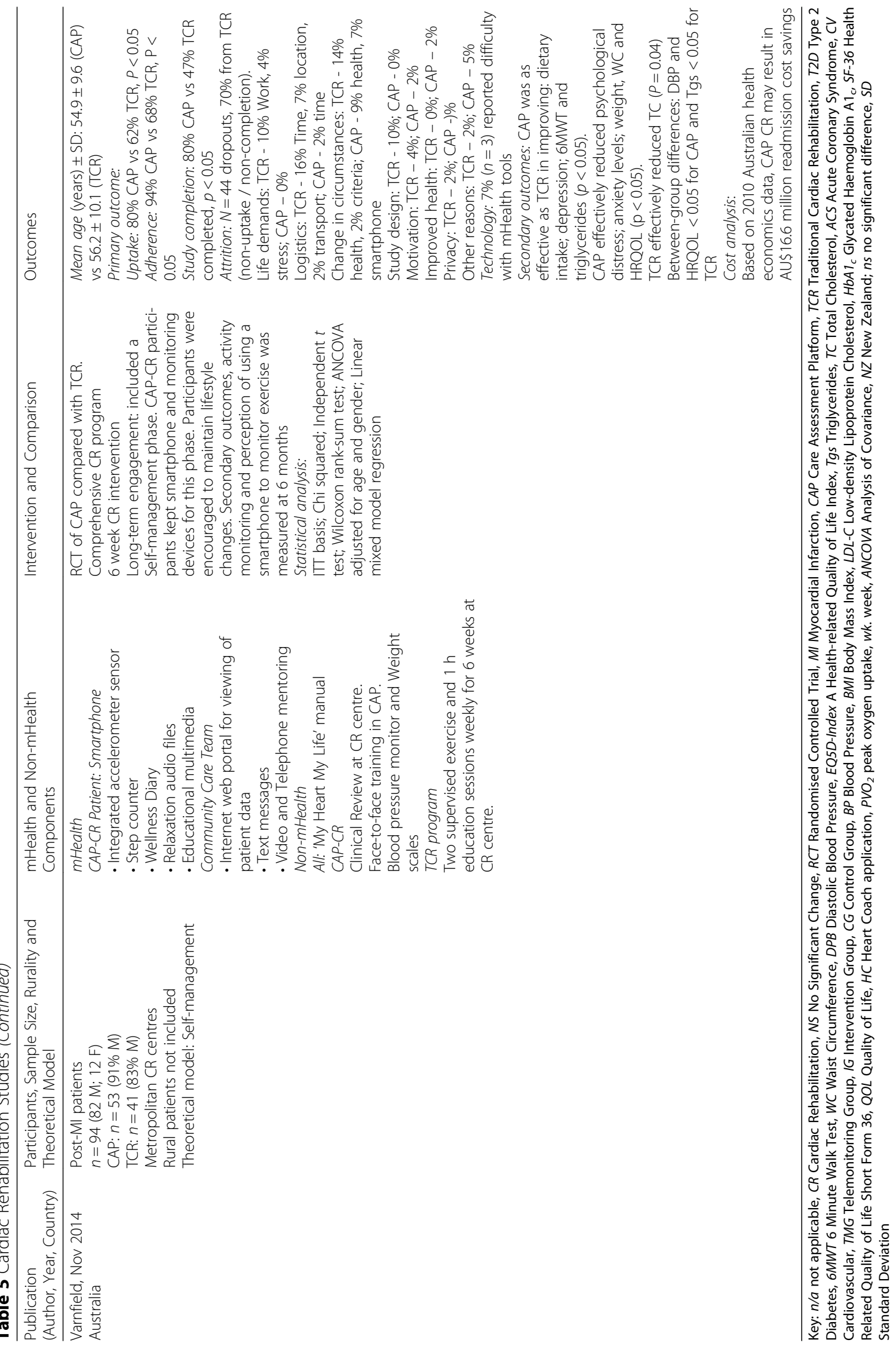


Table 6 Levels of evidence and outcome measures in Heart Failure studies

\begin{tabular}{|c|c|c|c|c|}
\hline & Scherr, 2006 & Scherr, 2009 & Seto, 2012 & Vuorinen 214 \\
\hline Country of Origin & Austria & Austria & Canada & Finland \\
\hline \multicolumn{5}{|l|}{ Levels of evidence } \\
\hline Design & FUU & $\mathrm{RCT}$ & $\mathrm{RCT}$ & $\mathrm{RCT}$ \\
\hline Level of Evidence* & IV & $\|$ & $\|$ & $\|$ \\
\hline CASP score ${ }^{* *}$ & $\mathrm{n} / \mathrm{a}$ & 6 & 8 & 8 \\
\hline Theoretical framework & & & $\checkmark$ & \\
\hline \multicolumn{5}{|l|}{ Outcome measures } \\
\hline \multicolumn{5}{|l|}{ Technology } \\
\hline Reliability & $\checkmark$ & & & \\
\hline Feasibility & $\checkmark$ & & & \\
\hline Clinical utility & $\checkmark$ & & $\checkmark$ & \\
\hline Usability & $\checkmark$ & $\checkmark$ & & $\checkmark$ \\
\hline Acceptability & $\checkmark$ & & & $\checkmark$ \\
\hline Adherence & $\checkmark$ & $\checkmark$ & $\checkmark$ & $\checkmark$ \\
\hline Usage & $\checkmark$ & $\checkmark$ & $\checkmark$ & $\checkmark$ \\
\hline Task completion & $\checkmark$ & & & \\
\hline \multicolumn{5}{|l|}{ Patient } \\
\hline Patient satisfaction & $\checkmark$ & & & $\checkmark$ \\
\hline Qualitative feedback & & & $\checkmark$ & $\checkmark$ \\
\hline $\mathrm{QOL}$ & & & $\checkmark$ & \\
\hline Self-care & & & $\checkmark$ & $\checkmark$ \\
\hline NYHA class & $\checkmark$ & $\checkmark$ & $\checkmark$ & \\
\hline LVEF & $\checkmark$ & $\checkmark$ & $\checkmark$ & $\checkmark$ \\
\hline $\mathrm{BP}$ and $\mathrm{HR}$ & $\checkmark$ & $\checkmark$ & $\checkmark$ & \\
\hline Weight & $\checkmark$ & $\checkmark$ & $\checkmark$ & \\
\hline ECG & & & $\checkmark$ & \\
\hline Medication use & $\checkmark$ & $\checkmark$ & $\checkmark$ & \\
\hline Biochemistry & & & $\checkmark$ & $\checkmark$ \\
\hline BNP & & & $\checkmark$ & $\checkmark$ \\
\hline Mortality & & $\checkmark$ & $\checkmark$ & $\checkmark$ \\
\hline Health service utilization & & $\checkmark$ & $\checkmark$ & $\checkmark$ \\
\hline Economic evaluation & \multicolumn{4}{|c|}{ None of these smartphone heart failure studies included a health economic analysis } \\
\hline
\end{tabular}

Key: $\checkmark$ Outcome measured, FUU Feasibility, Utility and Uptake study, RCT Randomised Control Trial, $n / a$ Not applicable, CASP Critical Appraisal Skills Programme, QOL Quality of life, NYHA New York Heart Association, LVEF Left ventricular ejection fraction, BP Blood pressure, HR Heart rate, ECG Electrocardiogram, BNP Brain Natriuretic Peptide

Participant engagement based on daily access was high [35], with usage [31] and session or task completions greater than $70 \%[30,35]$, and ease of use was high $(4.8 /$ 5) [30] (Tables 3 and 4). Qualitative feedback from participants and mentors [31] indicated an overall positive experience [35] and smartphone features were practical and easy to use [31] with a low frequency of minor technical problems [30]. However, Varnfield and colleagues reported that the Wellness Diary Connected internet portal was not regularly used by many participants (36\%) due to lack of computer or internet access [31].

\section{Uptake and utility}

Uptake of mHealth CR was assessed through enrolment, engagement in, acceptance of and adherence to the program with all three studies reporting on at least three of these parameters. Overall, CR delivered by mHealth was well accepted, with good participant enrolment (8693\%) [30, 35], daily engagement $(90 \%)[31,35]$, and adherence and task completion rates (78-91\%) [30, 31, 35] (Tables 3 and 4).

All three studies demonstrated mHealth effective for delivering the core components of $\mathrm{CR}[30,31,35]$. 
Table 7 Summary of outcome results in Heart Failure studies

\begin{tabular}{|c|c|c|c|c|}
\hline & $\begin{array}{l}\text { Scherr, } 2006 \\
\text { (FUU) }\end{array}$ & $\begin{array}{l}\text { Scherr, } 2009 \\
\text { (RCT) }\end{array}$ & $\begin{array}{l}\text { Seto, } 2012 \\
\text { (RCT) }\end{array}$ & $\begin{array}{l}\text { Vuorinen } 2014 \\
(\mathrm{RCT})\end{array}$ \\
\hline$n=$ & $\begin{array}{l}20 \\
14 \text { CHF; } 6 \text { HTN }\end{array}$ & $\begin{array}{l}120 \\
66 \text { TMG ( } 54+12 \text { never } \\
\text { beginners) } \\
54 \text { CG }\end{array}$ & $\begin{array}{l}100 \\
50 \text { TMG; } 50 \text { SCG }\end{array}$ & $\begin{array}{l}94 \\
(47 \mathrm{TMG} ; 47 \mathrm{CG})\end{array}$ \\
\hline Program completion & $\begin{array}{l}95 \%(n=19 \text { of } 20) \\
\text { CHF: } 93 \%(n=13 \text { of } 14) \\
\text { HTN: } 100 \%\end{array}$ & $\begin{array}{l}87 \%(n=104 \text { of } 120) \\
\text { TMG: } 76 \% \text { ( } n=50 \text { of } 66 \text {, includes } \\
\text { never beginners) or } 93 \%(n=50 \\
\text { of } 54) \\
\text { CG: } 100 \%\end{array}$ & $\begin{array}{l}97 \% \text { ( } n=97 \text { of } \\
\text { 100) } \\
\text { TMG: } 88 \% \text { ( } n=44 \\
\text { of } 47, \text { includes } 3 \\
\text { deaths) } \\
\text { or } 94 \% \text { ( } n=47 \text { of } \\
\text { 50, excludes } 3 \\
\text { deaths) } \\
\text { SCG: } 100 \%\end{array}$ & $\begin{array}{l}99 \%(n=1 \text { of } 94) \\
\text { TMG: } 98 \%(n=1 \text { of } 47) \\
\text { CG: } 100 \%\end{array}$ \\
\hline Mean age (years) & $\begin{array}{l}50 \text { (SD14) } \\
\text { CHF: } 53 \text { (13); HTN: } 42 \text { (16) }\end{array}$ & $\begin{array}{l}66 \text { (IQR 64-74) } \\
\text { TMG: } 66 \text { years (IQR 62-73) CG: } \\
67 \text { years (IQR 61-72) }\end{array}$ & $\begin{array}{l}\text { TMG: } 55.1 \pm 13.7 \\
\text { SCG: } 52.3 \pm 13.7\end{array}$ & $\begin{array}{l}\text { TMG: } 58.3 \pm 11.6 \\
\text { CG: } 57.9 \pm 11.9\end{array}$ \\
\hline \multicolumn{5}{|c|}{ Technology (Reported inconsistently) } \\
\hline Feasibility & $\checkmark$ high & & & \\
\hline Usability/Acceptability & $\begin{array}{l}80 \% \text { did not report any } \\
\text { problems with data entry }\end{array}$ & $98 \%$ system availability & & $\begin{array}{l}\text { 10-20 min initial education on use } \\
\text { of mobile phone app. } \\
\text { TMG: } 1 \text { patient withdrew due to } \\
\text { increased anxiety from monitoring } \\
\text { his condition. }\end{array}$ \\
\hline Technical & $\begin{array}{l}98 \% \text { data transmission and } \\
\text { website availability } \\
\text { One (5\%) withdrawl (poor } \\
\text { vision) }\end{array}$ & $\begin{array}{l}12 \text { never beginners (median age } \\
68 \text { years (IQR 64-74) were unable } \\
\text { to begin transmission of data } \\
\text { (reasons NR) }\end{array}$ & $\begin{array}{l}\text { TMG: } 2 \\
\text { participants } \\
\text { withdrew due to } \\
\text { technical } \\
\text { difficulties }\end{array}$ & $\begin{array}{l}\text { TMG: } 6 \text { telephone calls re technical } \\
\text { problems. } \\
3 \text { nurse initiated calls for start-up } \\
\text { support; } \\
3 \text { patient calls initiated for failed } \\
\text { internet connection. }\end{array}$ \\
\hline $\begin{array}{l}\text { Engagement/ } \\
\text { Adherence/ Usage/ } \\
\text { Task completion }\end{array}$ & $\begin{array}{l}\text { 94\% (CHF) and 84\% (HTN) } \\
\text { self-measurement and data } \\
\text { entry }\end{array}$ & 95\% patient adherence & $\begin{array}{l}\text { Completion of } \\
\text { daily readings: } \\
84 \% \text { completed } \\
50 \% \text {; } \\
66 \% \text { completed } \\
80 \% \text {; } \\
32 \% \text { completed } \\
95 \% \text {. }\end{array}$ & $\begin{array}{l}\text { Proportion of weekly submitted self- } \\
\text { measurements by TMG: } \\
86 \% \text { weight (median = } 28 \text { (IQ 23-33); } \\
89 \% \text { BP, HR, and symptoms (median } \\
\text { BP and symptoms = } 32 \text { (IQR 27-43). }\end{array}$ \\
\hline
\end{tabular}

Patient

Patient satisfaction

$85 \%$ of patients continued telemonitoring at study completion
$96 \%$ responded to user experience questionnaire

$95 \%$ - measures very or quite useful $91 \%$ - automatic feedback very or quite useful ( $9 \%$ no benefit) $66 \%$ - feedback drew attention to essential issues of disease 91\% - feedback was motivational

キ overall MLHFQ

\# Physical

f Emotional ++ Overall change $(p=0.05)$

Self-care

New York Heart Association class

Study completion vs baseline ++ (PPA)

Class I: $n=3$ vs 0

Class II: $n=11$ vs 10

Class III: $n=0$ vs 4

Left Ventricular Ejection Fraction
PPA: ns improvement
₹ † Maintenance

キ + Management ++ Maintenance

$\neq+$ 
Table 7 Summary of outcome results in Heart Failure studies (Continued)

\begin{tabular}{|c|c|c|c|c|}
\hline & $\begin{array}{l}\text { Scherr, } 2006 \\
\text { (FUU) }\end{array}$ & $\begin{array}{l}\text { Scherr, } 2009 \\
(\mathrm{RCT})\end{array}$ & $\begin{array}{l}\text { Seto, } 2012 \\
\text { (RCT) }\end{array}$ & $\begin{array}{l}\text { Vuorinen } 2014 \\
\text { (RCT) }\end{array}$ \\
\hline & $\begin{array}{l}\uparrow \text { in mean to } 35 \% \text { at study } \\
\text { completion (vs 32\% at } \\
\text { baseline) }\end{array}$ & $\begin{array}{l}\text { TMG: } 25 \%(\text { IQR } 20-38) \text { to } 35 \% \\
\text { (IQR 25-45) } \\
\text { CG: } 29 \%(\text { IQR } 21-36) \text { to } 35 \%(I Q R \\
24-40)\end{array}$ & & \\
\hline Blood Pressure & $\begin{array}{l}\text { HTN: mean study completion } \\
\text { SBP } 135 \text { (SD18); DPB } 78 \text { (7) vs } \\
\text { baseline SBP 134 (21); DBP } 80 \\
\text { (8) }\end{array}$ & & & \\
\hline Medication & $\begin{array}{l}\text { CHF: } 71 \% \text { had beta-blocker } \\
\text { therapy initiated with a } \\
\text { titrated increase } \\
\text { HTN: antihypertensive } \\
\text { medication stable }\end{array}$ & & $\begin{array}{l}\text { ₹ Aldosterone } \\
\text { antagonists }\end{array}$ & $\begin{array}{l}++ \\
\text { Medication change, both increases } \\
\text { and decreases. }\end{array}$ \\
\hline Biochemistry & & & & $\begin{array}{l}\text { ns } \\
\text { Serum potassium, creatinine, sodium. }\end{array}$ \\
\hline $\begin{array}{l}\text { Brain Natriuretic } \\
\text { Peptide }\end{array}$ & & & $\neq \dagger$ & $\begin{array}{l}\mathrm{ns} \\
\neq\end{array}$ \\
\hline $\begin{array}{l}\text { Mortality/Health } \\
\text { service utilization }\end{array}$ & & $\begin{array}{l}\text { ITT: ns } \\
\text { TMG: } 17 \% \text { ( } 0 \text { deaths/11) } \\
\text { hospitalizations } \\
\text { CG: } 31 \% \text { ( } 1 \text { death/17 } \\
\text { hospitalizations) } \\
\text { PPA: ++ } \\
\text { TMG: } 15 \% \text { (0 deaths/8 } \\
\text { hospitalizations) } \\
\text { PPA: ++ shorter length of } \\
\text { hospital stay }\end{array}$ & $\begin{array}{l}\text { TMG: } 6 \%(\mathrm{n}=3) \\
\text { deaths }(2 \text { non- } \\
\text { heart related) } \\
\text { SCG: } 0 \text { deaths } \\
\text { ns hospital } \\
\text { admissions; nights } \\
\text { in hospital; ED } \\
\text { visits } \\
\text { ++ Heart Function } \\
\text { Clinic visits (TMG: } \\
\text { 3.5 (SD 3.6); SCG } \\
2.5 \text { (2.5) }\end{array}$ & $\begin{array}{l}\text { No mortality in TMG or CG } \\
\text { ns HF related hospital days } \\
\text { ++ TMG nurse time, telephone } \\
\text { contact and visits } \\
\text { ++ TMG unplanned clinic visits } \\
\text { ++ patient initiated telephone } \\
\text { contact } \\
\text { ns physician time and visits }\end{array}$ \\
\hline
\end{tabular}

Key: FUU Feasibility, Utility and Uptake study, RCT Randomised Control Trial, CHF Chronic Heart Failure, HTN Hypertension, TMG Telemonitoring Group, SCG Standard Care Group, CG Control Group, QOL Quality of life, SBP Systolic Blood Pressure, DBP Diastolic Blood Pressure, ECG Electrocardiogram, ED Emergency Department, $N R$ not reported

FUU studies - pre- compared with post-intervention: + = significant improvement with $\mathrm{mHealth}$; $=$ no significant improvement with $\mathrm{mHealth}$; NR = not reported; $\uparrow=$ increased; $\checkmark=$ reported as feasible

RCT studies: $++=$ significant improvement in TMG compared with SCG (or CG); ns = no significant difference between TMG and SCG (or CG);

$\neq=$ significant improvement in SCG (or CG) compared with TMG; $\neq=$ significant TMG within-group differences at 6 months; $\dagger=$ significant SCG (or CG) withingroup differences at 6 months; ITT = Intention to treat analysis: PPA = Per Protocol Analysis

Worringham et al. demonstrated significantly improved physical function (6MWT) and Quality of Life (QOL) (SF36 Physical Health Score) and a reduction in depression, although there was no significant change on the SF36 QOL mental health scale (Tables 3 and 4) [30]. Forman et al. reported that the Heart Coach application resulted in $42 \%$ lower visit cancellations and improved participant adherence. [35]. Cardiac Rehabilitation staff reported an overall positive impact on their ability to provide quality $\mathrm{CR}$ care by enabling them to better anticipate and address issues as they occurred. CR staff feedback included: "Allowed clinicians to connect with individuals who could not attend CR"; "increased educational class attendance"; "enhanced patient participation in CR activities and increased accountability in CR activities at home" (Tables 3 and 4) [35]. Varnfield et al. reported that both Care Assessment Platform (CAP) CR and phone consultations with mentors motivated participants to achieve their rehabilitation goals [31]. While mentors highlighted the benefits of CAP CR for patients, they expressed concerns over the lack of exercise supervision and group support (Tables 3 and 4) [31].

\section{Randomised controlled trials}

Two RCTs identified are shown in Tables 3 and 4. Both studies were of similar study design; single-blind [36] or unblinded [32], parallel, two-arm RCTs. Blasco et al. also stratified by the presence of diabetes [36]. Follow-up periods were 6 and 12 months for the Varnfield and Blasco studies respectively.

\section{Participants}

Patients were included if they had a diagnosis of ACS and at least one coronary risk factor [36] or were postMyocardial Infarction patients referred to CR (Tables 3 and 4) [32]. Participants were middle aged and the majority were male $[32,36]$. Rural participants were not identified, and neither study reported on participant ethnicity or Indigenous status (Table 4) [32, 36]. 
Table 8 Heart Failure Studies

\begin{tabular}{|c|c|c|}
\hline $\begin{array}{l}\text { Publication } \\
\text { (Author, Year, } \\
\text { Country) }\end{array}$ & $\begin{array}{l}\text { Participants, Sample Size, } \\
\text { Rurality and Theoretical } \\
\text { Model }\end{array}$ & $\begin{array}{l}\text { mHealth and Non-mHealth } \\
\text { Components }\end{array}$ \\
\hline $\begin{array}{l}\text { Scherr, } 2006 \\
\text { Austria }\end{array}$ & $\begin{array}{l}\text { Patients with chronic Heart } \\
\text { Failure CHF or hypertension } \\
\text { (HTN) } \\
n=20(95 \% \mathrm{M}) \\
\text { CHF: } n=14(93 \% \mathrm{M}) \\
\text { HTN: } n=6(83 \% \mathrm{M}) \\
\text { Metropolitan and rural } \\
\text { patients } \\
\text { Theoretical model: None } \\
\text { Comparison group: None }\end{array}$ & $\begin{array}{l}\text { mHealth } \\
\text { Patient terminal: Mobile } \\
\text { phone with Wireless } \\
\text { Application Protocol (WAP) } \\
\text { technology } \\
\text { Physician's terminal: Personal } \\
\text { Computer with internet } \\
\text { access } \\
\text { Non-mHealth } \\
\text { Automatic BP monitor } \\
\text { Digital weight scales } \\
\text { Doctor-patient relationship } \\
\text { Patient completed } \\
\text { questionnaires on the } \\
\text { technical aspects of the } \\
\text { telemonitoring system }\end{array}$ \\
\hline
\end{tabular}

Scherr, 2009

Austria
Patients with heart failure and a hospital admission of $>24 \mathrm{~h}$ in the last 4 weeks. $n=120$ (66 TMG; 54 SCG)

TMG: $n=12$ never beginners (50\% M)

TMG: $n=54(74 \% \mathrm{M})$

CG: $n=54(72 \% \mathrm{M})$

Metropolitan centres

Rural patients not reported.

Theoretical model: None
mHealth

Mobile phone with Wireless

Application Protocol (WAP)

technology, Weight scale and automated BP monitor Secure web-based CRF at monitoring centre.

Patients measured BP, HR and weight daily.

Data entered and automatically sent to the remote server at remote Email alerts to study physician Study physician accessed data and could phone patient on mobile
Intervention

Observational study to

evaluate acceptability,

feasibility and reliability of a

telemonitoring system.

90 day follow-up

Long-term engagement: no engagement or follow-up reported beyond the 90 day

intervention period. Patients measured BP, HR and weight daily and transferred data via mobile phone.

Physician automatically notified by SMS of any parameters outside a pre-set range.

Study physician accessed data and phoned patient as necessary for therapeutic adjustments

Automatic reminders set by study physician

Statistical analysis: Descriptive statistics reported.

Prospective, open-label RCT TMG: Pharmacological treatment with telemedical surveillance

CG: pharmacological treatment alone and no planned interaction with study site.

6 months follow-up Long-term engagement: no engagement or follow-up reported beyond the 6 month intervention period.

Statistical analysis: Per protocol principle and intention to treat analysis. Log-rank test, Kaplan-Meier estimation and relative risk
Outcomes

Mean Age: All, 50 (SD14) years; CHF, 53 (SD13) years; HTN, 42 (SD 16) years. Study completion/attrition: 95\% ( $n=19)$ completed CHF: $93 \%(n=13)$ completed HTN: $100 \%$ ( $n=6$ completed) $5 \%(n=1)$ withdrew from

TMG due to poor vision Reliability

98\% data transmission success

98\% website availability for physicians.

Feasibility and Acceptability: Implausible data entry: 5 per CHF patient; 4 per patients with HTN

Successful transmissions: 83\%

CHF and 84\% HTN

Self-measurement and data entry: CHF - 85 data transfer sessions over 90 days;

HTN - two BP and HR measures on an average of 453 out of 540 cumulative days

Patient acceptance

High, 17 patients continued with telemonitoring at study end.

Study completion/attrition: 19 participants completed. One participant withdrew due to inability to operate the mobile phone because of poor vision.

Clinical utility

CHF patients: stable or improved: mean LVEF improved from 32\% to 35\% Beta-blocker initiation supported: commenced and up titrated successfully in 10 of the 14 CHF patients Patients with HTN: BP stable; $134 / 80 \mathrm{mmHg}$ at baseline vs $135 / 78 \mathrm{mmHg}$ at completion

Median age:

12 never beginners 68 years (IQR 64-74);

TMG 65 years (IQR 62-72); CG 67 years (IQR 61-72)

Study completion/attrition: 104 participants completed. 12 participants were unable to transmit data - classified as never beginners.

4 TMG participants withdrew early (included in intentionto-treat and per-protocol analysis).

Participant adherence: 95\% Intention-to-treat analysis: TMG vs CG: 0 deaths and 11 hospitalizations (17\%) vs 1 
Table 8 Heart Failure Studies (Continued)

Publication Participants, Sample Size,

(Author, Year, Rurality and Theoretical

mHealth and Non-mHealth

Intervention

Outcomes

Country) Model

Seto, 2012

Canada
Heart Failure patients with

LVEF $<40 \%$

$n=100$

TMG: $n=50(82 \% \mathrm{M})$

SCG: $n=50(76 \% \mathrm{M})$

Metropolitan centre (possible patients from rural or remote settings)

Theoretical model: Self-care

\author{
Study physician could set \\ automatic reminders. \\ Non-mHealth \\ CG: Pharmacological care
}

reduction utilized for primary endpoint. Secondary endpoints: $t$-test, chi-square test, Wilcoxon rank sum test and Wilcoxon signed rank test utilized.
mHealth

TMG: Smartphone with blue tooth capability, BP monitor and scales; ECG recorder provided to 17 TMG participants - data automatically sent wirelessly to data repository.

Daily morning symptom questions

Email and text messages Website viewing of results by clinicians and patients. Non-mHealth

All

Pre and post study:

Demographic, clinical data,

SCHFI and MLHFQ

questionnaires

SCG:

Clinic visits

Optimization of medication Heart Failure education

Telephone contact

TMG:

Standard care as per SCG
Non-blinded RCT

TMG vs Standard Care Group (SCG)

Stratified 4 block

randomization based on

NYHA classification.

6 month follow-up:

post-study questionnaire; 22

semi-structured interviews

with TMG participants; 5

semi-structured interviews with clinicians

Long-term engagement: no engagement or follow-up

reported beyond the 6 month intervention period.

SCG participants were not contacted by the study site until study end.

Statistical analysis: Between group analysis: Student t tests and Mann-Whitney tests. Within group analysis: Paired Student $t$ tests and Wilcoxon signed rank tests. death and 17 hospitalizations (33\%), a RRR of $50 \%(95 \%$ Cl $3-74 \%), p=0.06$

TMG: majority of rehospitalizations occurred in first month of follow-up.

Per protocol analysis: TMG: 0 deaths and 8 hospitalizations (15\%), a RRR of $54 \%(95 \% \mathrm{Cl} 7-79 \%)$, $p=0.04$

NYHA class improved (III to II) in TMG, $P<0.001$ vs CG and TMG baseline Median length of stay: TMG 6.5 vs SCG 10.0 days (IQR 7.0-13), $p=0.04$

LVEF: ns improvement in both TMG and CG. TMG 25\% (IQR 20-38) to 35\% (IQR 2545) and CG 29\% (IQR 21-36) to $35 \%$ (IQR 24-40)

375 alerts, 170 contacts, 55 adjustments to heart failure medications.

Mean age: TMG 55.1 years (SD 13.7); SCG 52.3 years (13.7)

Completion/attrition: 97 participants completed. 3 participants withdrew from TMG (1 was incapacitated after a fall; 2 because of technical difficulties). No participant withdrew from SCG.

Patient adherence: 84\%, 66\% and $32 \%$ completed at least $50 \%, 80 \%$ and $95 \%$ of possible daily readings. Health service utilization: no significant differences in hospital admissions; nights in hospital; and ED visits. Number of Heart Function Clinic visits increased in TMG $(p=0.04)$ due to unplanned cardiologist recalls in response to telemonitoring system alerts.

Improvement post-study for TMG and SCG - BNP values $(p=0.001$ and $p=0.002)$; NYHA class ( $p=0.000$ and $0.001) ; \operatorname{LVEF}(p=0.001)$; and self-care $(p=0.004$ and $P=0.006)$. QOL improved only in the TMG $(p=0.02)$. Between group post-study only self-care maintenance (SCHFI) was significant $(p=0.03)$. Between group change - only overall QOL (MLHFQ) $(p=0.05)$ 
Table 8 Heart Failure Studies (Continued)

\begin{tabular}{|c|c|c|}
\hline $\begin{array}{l}\text { Publication } \\
\text { (Author, Year, } \\
\text { Country) }\end{array}$ & $\begin{array}{l}\text { Participants, Sample Size, } \\
\text { Rurality and Theoretical } \\
\text { Model }\end{array}$ & $\begin{array}{l}\text { mHealth and Non-mHealth } \\
\text { Components }\end{array}$ \\
\hline $\begin{array}{l}\text { Vuorinen, 2014, } \\
\text { Finland }\end{array}$ & $\begin{array}{l}\text { Heart Failure patients with } \\
\text { LVEF } \leq 35 \%, \text { NYHA class } \geq 2 \\
n=94 \\
\text { TMG: } n=47 \text { ( } 83 \% \mathrm{M}) \\
\text { CG: } n=47 \text { ( } 83 \% \mathrm{M}) \\
\text { Metropolitan centre (possible } \\
\text { patients from rural or remote } \\
\text { settings) } \\
\text { Theoretical model: none }\end{array}$ & $\begin{array}{l}\text { mHealth } \\
\text { Patient: Mobile phone with } \\
\text { preinstalled software app } \\
\text { Provided with weight scale, } \\
\text { blood pressure monitor, } \\
\text { mobile phone with app and } \\
\text { self-care instructions. } \\
\text { Non-mHealth } \\
\text { Multidisciplinary clinic visits } \\
\text { and nurse feedback by } \\
\text { telephone. }\end{array}$ \\
\hline
\end{tabular}

Intervention Outcomes

Country)

LVEF $\leq 35 \%$, NYHA class $\geq 2$

TMG: $n=47(83 \% \mathrm{M})$

CG: $n=47(83 \%$ M)

( settings)

Theoretical model: none
Prospective RCT
TMG vs usual care (CG)

6-month follow-up

Long term engagement: no engagement or follow-up reported beyond the 6month intervention period TMG:

Patients evaluated $\mathrm{BP}, \mathrm{HR}$, weight, symptoms and change in overall condition, weekly and transferred data via mobile phone application Received by secure remote patient monitoring server Patients received automated feedback about whether reported data was within personal targets set by nurse. Nurses accessed data and phoned patient weekly or as necessary for out-of-target parameters or failure to upload data

CG:

Patients encouraged to measure weight, blood pressure and heart rate at home

Cardiac team monitor and interpret symptoms, optimize medication and provide education

Statistical analysis: ZIP regression used for outcome variables that expressed counts, contiguous variables analysed within and between study groups

\author{
Mean age: TMG 58.3 (SD \\ 11.6) CG 57.9 (11.9) \\ Completion/attrition: 1 patient \\ from TMG lost to follow-up. \\ Patient adherence \\ $=$ proportion of weekly \\ submitted self-measurements \\ by TMG: \\ $86 \%$ weight $($ median $=28$ \\ (IQR 23-33), \\ $89 \% \mathrm{BP}, \mathrm{HR}$, and symptoms
}

(median BP and

symptoms $=32($ IQR 27-43)

Feasibility and Acceptability: 96\% (44/46) from TMG

responded to survey, 42/44

found making/reporting measurements with mobile app "useful" or "very useful."

91\% automatic feedback very or quite useful $(9 \%$ no benefit),

$66 \%$ feedback drew attention to essential issues of disease, 91\% feedback was motivational.

Primary Outcome: Mean HFrelated hospital days: 0.7 (TMG) vs 1.4 (CG) $(p=0.351)$ Secondary Outcomes:

Clinical: change in NTproBNP, LVEF \%, EHFSBS score, serum creatinine, potassium and sodium not significantly different between groups. Mortality: 0 (control), 0 (TMG).

Within group changes were significant for: LVEF increased $5.0 \%, p=0.003$ TMG and $4.2 \%, p=0.001$ CG; EHFSBS ( -5.0 points, $p<0.001$ TMG and $-3.8, p<0.001)$; NTproBNP decreased in the TMG $(-198 \mathrm{ng} / \mathrm{l}, p=0.01)$ Use of health care resources: Mean nurse time, telephone contacts and visits higher in TMG $(p<0.001)$;

TMG unplanned visits to Cardiac Outpatient Clinic higher $(p<0.001)$;

TMG patient initiated telephone contact higher $(p<0.049)$;

No statistical difference between groups for physician time and visits.

Key: CHF Chronic Heart Failure, n/a not applicable, RCT Randomised Controlled Trial, TMG Telemonitoring Group, SCG Standard Care Group, CG Control Group, SCHFI Self-Care of Heart Failure Index, MLHFQ Minnesota Living With Heart Failure, NYHA New York Heart Association, LVEF Left Ventricular Ejection Fraction, BNP Brain Natriuretic Peptide, ED Emergency Department, QOL Quality of Life, ECG Electrocardiogram, BP Blood Pressure, HR Heart Rate, CV Cardiovascular, CRF Case Report Form, ED Emergency Department, NT-proBNP N-terminal of the prohormone brain natriuretic peptide, EHFSBS European Heart Failure Self-Care Behaviour Scale 


\section{Interventions}

Two distinct intervention approaches have been utilized and are summarised in Table 4 [32, 36]. Blasco et al. utilized telemedicine as an adjunct to lifestyle counselling and usual care during a 12-month followup period with the aim of assessing the efficacy of the telemedicine system [36]. Varnfield and colleagues undertook a study aimed at examining whether CAPCR was effective at improving $C R$ use and health outcomes compared with traditional centre-based CR programs, and addressed all components of a comprehensive CR program via mHealth delivery in a RCT [32]. The CAP CR platform was downloaded onto a smartphone and provided health and exercise monitoring, delivery of motivational and educational information via text messages, and preinstalled audio and video files according to weekly themes [32].

\section{Acceptability}

The acceptability of mHealth $\mathrm{CR}$ was assessed by the number of sessions completed [36] or uptake, adherence and completion rates [32] and was demonstrated to be high in both studies (Tables 3 and 4). Varnfield et al. demonstrated significant increases in uptake, adherence and program completion compared with the TCR group (Tables 3 and 4). A small number of participant's reported difficulty with using the mHealth tools [32] with low numbers of withdrawals occurring because of participant stress related to technology use or their inability to handle the technology [36] (Tables 3 and 4) .

\section{Outcomes}

The efficacy of CR delivered by mHealth (smartphone \pm usual care and/or other eHealth methods) was as effective as or exceeded for some parameters that of traditional centre-based CR or usual care. Outcomes are shown in Tables 3 and 4 and outcome measures in Table 2 .

On the basis of intention to treat analysis, Blasco et al. reported that the tele-monitoring group were significantly more likely compared with the control group (RR $=1.4 ; 95 \% \mathrm{CI}=1.1-1.7$ ) to achieve the primary outcome of cardiovascular risk improvement (defined as the proportion of patients who achieved the goal of treatment in at least one cardiac risk factor without exacerbation of any of the others) and treatment goals for blood pressure (Tables 3 and 4) [36]. There was no significant between-group difference for smoking cessation or LDLC. The tele-monitoring group achieved significant changes in all outcome measures $(p<0.05)$ with the exception of diastolic blood pressure; the control group achieved significant changes in diastolic blood pressure $(p=0.001)$ [36].
In the Varnfield et al. study, the primary outcomes of uptake, adherence and completion were 1.3, 1.4 and 1.7 times more likely in CAP-CR compared with traditional centre-based rehabilitation (TCR) [32]. Both CAP-CR and TCR were effective at improving the secondary outcomes from baseline to 6-week follow-up and betweengroup changes from baseline to 6-weeks were similar for both groups, with the exception of diastolic blood pressure and health related QOL (EQ5D)-index for CAP-CR and triglycerides for TCR [32] (Tables 3 and 4). An assessment of cost-effectiveness based on 2010 Australian health economics data suggested that increased CR completion rates with fewer admissions and deaths would result in AU\$16.6 million readmission cost savings [32].

Table 3 reports a comparative summary of outcomes for the CR studies. Of the outcomes that were reported for both RCT studies, only quality of life was significantly improved in the mHealth interventions compared with control groups [32, 36]. Systolic and diastolic blood pressure, Haemoglobin $\mathrm{A} 1_{\mathrm{c}}$ and plasma lipid levels (LDL-c and triglycerides) were reported in both studies with inconsistent outcomes [32, 36].

\section{Heart failure studies}

Four studies focused on improving outcomes through use of mHealth in heart failure rehabilitation and disease management: one feasibility, utility and uptake study, and three RCTs [37-39] (Tables 4, 5 and 7). Scherr et al. did not directly report on the inclusion of rural participants but did report poor reception of mobile phones in rural areas $[37,38]$. Seto et al. included participants from metropolitan and possibly rural settings as indicated by the statement that some patients needed to travel a number of days prior to arriving home [39]. Vuorinen et al. reported that the study was conducted in a metropolitan area and that patients did not have to travel far to obtain health services [40]. Only Seto et al. utilised a theoretical framework, that of self-care, with measurement based on the Self-Care of Heart Failure Index (SCHFI) [39].

\section{Feasibility, utility and uptake study}

Scherr et al. evaluated a newly developed telemedicine system for its acceptability, feasibility and reliability in supporting 14 patients (13 male) with heart failure and 6 (5 male) with hypertension in a 90 day observational study [37] (Tables 4, 5 and 7). Heart failure was defined as being symptomatic for at least six months, a mean left ventricular ejection fraction $<45 \%$, a resting heart rate $>$ 60 beats per minute and therapy with angiotensinconverting enzyme inhibitors. The telemedicine system integrated care through a mobile phone, a physician website via a personal computer and a server and participants were provided with an automatic blood pressure 
monitor and a digital weight scale for daily use. Data was entered into templates on the mobile phone and sent automatically to the server for monitoring by study physicians [37].

Overall, the reliability of server accessibility was high for both data transmission and website availability [37]. Poor access related to limited connectivity for mobile phones in rural areas accounted for unsuccessful data transmissions. The feasibility and acceptability of mHealth delivery for heart failure management was demonstrated. The level of implausible data entry was low and successful transmission, adherence with selfmeasurements and data entry were high over the 90 day monitoring period [37]. One dropout occurred due to an inability to operate the system because of low vision. Acceptability was high with only two reports of problems in reading the mobile phone display [37]. Data entry took approximately two minutes and was rated as acceptable. Patients also reported that electronic reminders improved their adherence to measurement and hence their awareness of body weight and blood pressure. Acceptability was further indicated by 17 patients continuing with monitoring after they completed the study [37].

The clinical status of patients with heart failure was stable or improved at study end, as indicated by mean left ventricular ejection fraction (LVEF). Telemonitoring also supported the initiation of beta-blocker therapy in patients with heart failure [37].

\section{Randomised controlled trials}

Three randomised controlled trials were identified (Tables 4, 5 and 7), all open-label (non-blinded) trials where participants were randomly allocated to standard care plus telemonitoring (TMG) or standard care alone (SCG) [38-40]. All three interventions took place over six months without longer-term engagement or followup beyond the six-month intervention period.

\section{Participants}

All studies included patients with a diagnosis of heart failure [38, 39]. Scherr et al. included patients with worsening heart failure (acute cardiac decompensation) and a hospital admission lasting $>24 \mathrm{~h}$ within the previous 4 weeks [38]. Seto et al. included patients with a LVEF < $40 \%$ and an expected survival of greater than a year [39]. Vuorinen et al. included patients with LVEF $\leq 35 \%$, and NYHA class of $\geq 2$ [40]. Patients were middle aged (mean TMG 55 years and SCG 52 years and TMG 57.9 and SCG 58.3 years, in Seto et al. and Vuorinen et al. respectively) compared with an older patient group (median 66 years) in the study by Scherr et al. [38-40]. Although Seto et al. reported on ethnicity, Indigenous status was not identified [39].

\section{Interventions}

All studies utilized a telemedical (mobile phone with smartphone functionality or Wireless Application Protocol (WAP) technology) surveillance system to monitor patient status in addition to standard care compared with standard care alone [38, 39]. Scherr et al. compared pharmacological treatment and telemedical surveillance with pharmacological treatment alone. The combined primary endpoint was cardiovascular mortality or re-hospitalization for worsening heart failure [38]. Participants entered their daily measures and heart failure medication dosage into the mobile phone and sent them to the monitoring centre for review by study physicians. Email alerts were sent to the study physician if transmitted data was outside of individually adjustable parameters or if there was a weight increase of greater than $2 \mathrm{~kg}$ in 2 days. If necessary, study physicians contacted the patient using their mobile phone [38].

Seto et al. compared telemonitoring in addition to standard care with standard care alone [39] using brain natriuretic peptide (BNP), self-care and QOL as the primary outcome measures. The study was underpowered to detect between group differences in hospital readmissions, number of nights in hospital and mortality, and hence these were secondary outcomes [39]. Standard care consisted of visits to a Heart Function Clinic, medication optimisation, heart failure education and the ability to contact the clinic as necessary. The TMG utilised the telemonitoring system (phone, BP monitor, weight scales and ECG recorder) for daily monitoring and data was sent automatically via Bluetooth to the mobile phone and then to a data repository for review by clinicians and participants. Participant and clinician experience with the system was examined by semistructured interviews [39].

Vuorinen et al. compared telemonitoring in addition to standard care against standard care alone [40]. Days spent in the hospital for heart failure was the primary outcome measure with multiple secondary outcomes including clinical outcomes, use of health care resources (mean time with nurse or physician, telephone contacts by nurse and by patient, visits to nurse, visits to physician, and unplanned visits to Cardiac Outpatient clinic) and patient experience [40]. Standard care consisted of self-measurement of $\mathrm{HR}, \mathrm{BP}$, and weight at home and regular visits to the cardiac clinic. Contact by telephone was added to standard care as necessary. The TMG utilized a mobile phone with a preinstalled software app for weekly monitoring of $\mathrm{HR}$, BP, weight, and symptoms. Data was sent to a secure patient server where it could be accessed by the cardiac team through a web-based user interface. Participant experience with the system was elicited by survey [40]. 


\section{Outcomes}

Telemonitoring for heart failure management was demonstrated to be feasible and acceptable with high patient adherence and to the potential to reduce hospital service utilization through lowering the frequency and duration of hospitalisations [38, 39] (Outcome measures are shown in Table 4 and outcomes in Tables 5 and 7). Utilizing a per-protocol analysis, Scherr et al. demonstrated a significant $54 \%$ relative risk reduction (RRR) of hospitalisation for the telemonitoring compared with control group and although intention-to-treat analysis did not reach significance (RRR 50\%, $p=0.06$ ) [38]. However, the benefit of telemonitoring was not evident within the first month of follow-up when the majority of hospital re-admissions occurred. Compared with the controls, the per-protocol analysis demonstrated those with telemonitoring had a significantly shorter length of hospital stay for those hospitalised for worsening heart failure [38]. Seto et al. demonstrated an increased rate of cardiologist review for deteriorating health status identified through telemonitoring although no difference in hospital service utilisation; however, this was not a primary outcome and the study was underpowered to detect a difference in these parameters [39]. Vuorinen and colleagues demonstrated a non-significant reduction in heart failurerelated hospital days in the telemonitoring group compared with the standard management (0.7 (SD 2.4) vs 1.4 (SD 3.5)). However, there was a significantly higher health care resource utilization in the telemonitoring group for mean nurse time, contacts and visits, cardiac outpatient clinic visits and patient initiated telephone contact but not physician time and visits [40].

In a per-protocol analysis, Scherr et al. demonstrated a significant improvement for New York Heart Association (NYHA) class and a non-significant improvement in left ventricular ejection fraction in those using telemonitoring [38]. While there were improvements demonstrated by Seto et al. in brain natriuretic peptide, NYHA class, LVEF, self-care maintenance and self-care management improved for both telemonitoring and standard management groups, physical and emotional QOL (measured by Minnesota Living with Heart Failure Questionnaire (MLHFQ)) were significantly improved for patients using telemonitoring [39]. Between-group post-study data analysis indicated that the group using telemonitoring had greater improvement in self-care maintenance and improvement in overall QOL (MLHFQ). Post-hoc subgroup analysis of 63 patients attending the Heart Failure clinic for greater than 6 months demonstrated significant improvement in BNP $(p=0.02)$, LVEF $(p=0.005)$, self-care maintenance $(p=0.05)$ and self-care management $(p=0.03)$ in the group using telemonitoring [39]. Vuorinen et al. demonstrated no significant improvements for NT-proBNP, LVEF, EHFSBS, and serum creatinine, potassium and sodium between groups [40]. However, in both groups there were significant improvements in LVEF and EHFSBS $(p<0.003)$ and a significant reduction in NT-proBNP with telemonitoring $(p=0.01)$. Medication adjustments, both increases and decreases, were significantly higher with telemonitoring compared with standard care [40].

\section{Discussion}

Despite the heterogeneity of the studies reviewed, mHealth was shown to be feasible with high rates of participant engagement, acceptance, usage and adherence. The efficacy of mHealth was comparable to traditional centre-based CR, however, reductions in hospital service utilization for heart failure patients was inconsistent. mHealth has the potential to be an effective method of delivering $\mathrm{CR}$ and heart failure management and improving access for patients unable to attend traditional centre-based rehabilitation programs, however, larger high quality studies are required for more definitive conclusions to be drawn.

For smartphones to be an effective platform for supporting behaviour change and self-management of health conditions, the smartphone needs to be a vital and inseparable aspect of the intervention [41]. Intervention designs informed by behaviour change theory are more effective than those without a theoretical base [42]. A behaviour change framework, the Fogg Behaviour Model, provides for a shared understanding of human behaviour that is useful in the analysis and design of persuasive technologies [43]. The Fogg Behaviour Model identifies and defines three principle factors that control whether a behaviour is likely to be performed; motivation, ability and triggers [43]. Oinas-Kukkonen extended Fogg's work by introducing a framework to classify technology in its persuasive functions, the persuasive system design [41, 44]. Persuasive systems are defined as "computerized software or information systems designed to reinforce, change or shape users' attitudes or behaviours without using coercion or deception" [41]. Published studies of mHealth delivery of $\mathrm{CR}$ to date have not specifically addressed behaviour change strategies in intervention designs $[45,46]$. However, a mHealth healthy-eating pilot study conducted by Dale et al. utilized a healthy eating intervention framed within social cognitive theory that resulted in a post-intervention increase in environmental self-efficacy when making food choices [47]. Behaviour change theories were limited to only 3 of 9 the studies in this review and included the theories of self-care [39] and a model of selfmanagement [31, 32]. 
The development, evaluation and implementation of complex interventions and innovative approaches for the delivery of health care requires an iterative program of research with a systematic approach [33, 34, 48-50]. Both iterative components to explore discovery, development and evaluation of effectiveness leading to implementation and applied programmes of research for mHealth home-based $\mathrm{CR}$ or heart failure management have been reported [33, 34, 48, 49, 51]. Scherr and colleagues and Varnfield et al. show an iterative program of development, evaluation and implementation through feasibility studies and RCTs [31, 32, 37, 38].

A framework for developing and evaluating mobile applications for CR suggests the following six principles: the core components of CR should be addressed; individual tailoring of features are enabled; behaviour change theory is applied; high usability is demonstrated; patient centred outcomes are improved; and efficacy in a randomized clinical trial is established [45]. Varnfield et al. is the only identified RCT that comprehensively addresses the core components of CR via mHealth [32]. These are documented in the internal feasibility study (Table 1 in [31]) and in the RCT (Fig. 1 in [32]) where CR components were delivered by text messages, preinstalled audio and video files and mentoring sessions. Participants were also provided with the National Heart Foundation "My Heart My Life" manual [52].

Two design principles in the primary task support category of the persuasive system design framework are tailoring and personalization [41]. Both relate to the persuasive capabilities of the system and are closely related. Tailoring relates to the potential needs, interests, personality, usage context, or other factors relevant to a user group, whereas personalization relates to personalized content or services [41]. All studies included in this review had features that enabled personalization of information delivery, most commonly through feedback of results by telephone, telephone/video mentoring and individualised text messages. A study by Antypas found that tailoring a mobile phone intervention to enhance maintenance of physical activity after CR utilizing SMS text messaging resulted in no difference in perceptions of personal relevance of the intervention although compared with the control group the tailored intervention group maintained a significantly higher level of physical activity at 3 months post discharge [53]. In a systematic review of adherence to web-based interventions, Kelders et al. examined whether intervention characteristics and persuasive design affected adherence. They reported that primary task support plays a more important role in the effect of an intervention with differences in technology and interaction predicting adherence [44].
No RCT in this review included a follow-up of longer than 12 months. Out of the five RCT studies included, four studies had an intervention or followup period of 6 months [32, 38-40], while one had a 12 month follow-up period [36]. Given the chronic nature of the conditions these interventions address, the lack of long-term follow-up leaves open to question their longer term effectiveness. Patient feelings of abandonment and difficulties integrating into community exercise programs after $C R$ have been reported [54], so how mHealth interventions fare warrants further study given the implications for the long-term maintenance.

Assessment of cost-effectiveness is an important component of pre-trial modelling [55] and evaluation of complex interventions $[48,56]$. A cost-effectiveness analysis was not formally undertaken in any of the studies included in this review, although a cost-estimate based on 2010 Australian health economic data for CAP CR [32] found a likelihood of cost-savings based on higher CR completion rates and reduced hospital admissions and mortality [32]. Health service utilization was reported in the three heart failure RCT's with inconsistent results. Significantly reduced hospitalisations and shorter length of hospital stay was reported in one study [38], whereas the other two studies reported no significant difference in heart failure related hospital days or admissions but a significant increase in Heart Function Clinic visits [39] or increased nurse time related to telephone contact (nurse and patient initiated) and unplanned clinic visits [40].

To date, evidence for mHealth effectiveness in CR or heart failure management has primarily included participants from metropolitan settings. There is a paucity of evidence for the adaptability and effectiveness of these programs in rural, remote and Indigenous patients, showing that further research is required [5]. Those studies reporting inclusion of rural patients [30, 37, 39] did not include subgroup analyses of outcomes for these patients, possibly due to a limited number of participants although periodic connectivity interruptions for rural participants were reported [30, 37]. This gap means that the feasibility of mHealth delivery of $\mathrm{CR}$ and heart failure management requires testing in rural and remote settings where non-centre based care is particularly needed. Implementation of mHealth in these settings requires robust evaluation.

This review included interventions utilizing smartphone functionality both through the use of a smartphone and through the use of mobile phones with Wireless Application Protocol (WAP) capabilities. Six studies used smartphones in their interventions, while three studies used mobile phones with WAP capabilities. While differences in outcomes for the two different 
types of smartphone functionality are not clear, the smartphone's educational and other support capabilities should be considered.

Restriction of the literature search to smartphone functionality is a strength of this review in a nascent and fast growing method of health care delivery. The search excluded other alternative methods of home-based CR and heart failure management as these have been reported elsewhere [5]. The search also excluded mHealth interventions that solely used SMS text messaging. mHealth studies using SMS text-messaging were excluded because this approach does not comprehensively address the core components of $\mathrm{CR}$, specifically exercise, education, and psychosocial support. SMS-based studies included interventions such as SMS goal setting and activity reminders and automated health promotion messages to promote exercise and smoking cessation $[53,57,58]$, but they were limited in the amount of education, feedback, and psychosocial support they could provide. $[53,57,58]$. It is possible that utilizing a combination of multiple technology modalities (smartphones, SMS text messaging and/or mentoring by phone) may prove superior to the use of a single modality such as smartphone use alone. Indeed, Varnfield et al., highlighted the importance of mentoring interactions via the mobile phone to motivate patients to achieve their goals [31]. Limiting the search to smartphone functionality has resulted in a small number of studies being eligible for inclusion in this systematic review.

Another limitation of the studies was the relatively small sample size of some studies and the limited follow-up times of the RCTs available. Given the studies with smaller sample sizes (6 to 26 participants) were feasibility, utility and uptake studies [30,31,37], their inclusion does not impact on the analysis of effectiveness of mHealth compared with TCR in the RCTs reported on [32, 36, 38-40].

\section{Conclusion}

mHealth delivery of $\mathrm{CR}$ and heart failure management is feasible with high rates of participant engagement, acceptance, usage and adherence. The efficacy of mHealth in these studies was comparable to traditional centre-based CR. mHealth delivery has the potential to improve access to $\mathrm{CR}$ and heart failure management for patients unable to attend traditional centre-based programs. The higher proportion of Indigenous people in more remote areas means that mHealth applications for particular subgroups needs special consideration. Feasibility testing of mHealth delivery for $C R$ and heart failure management for rural and remote settings in Australia should include assessment of cultural compatibility with careful evaluation of implementation for rurally based health services and consumers.

\section{Additional file}

Additional file 1: Search Strategies. This supplementary document details four database search strategies: Three for all databases searched except Medline and the fourth is for MEDLINE. (DOC $34 \mathrm{~kb}$ )

\section{Abbreviations \\ ACS: Acute Coronary Syndrome; BNP: Brain Natriuretic Peptide; CAP: Care Assessment Platform; CASP: Critical Appraisal Skills Programme; CHF: Chronic Heart Failure; CR: Cardiac rehabilitation; CVD: Cardiovascular disease; DBP: Diastolic Blood Pressure; ECG: Electrocardiogram; ED: Emergency Department; FUU: Feasibility, Utility and Uptake study; GPS: Global positioning system; $\mathrm{HbA}_{\mathrm{c}}$ : Haemoglobin $\mathrm{A} 1_{c} ; \mathrm{HTN}$ : Hypertension; \\ ICT: Information and communication technologies; LVEF: Left ventricular ejection fraction; mHealth: Mobile health; MLHFQ: Minnesota Living with Heart Failure Questionnaire; NHMRC: National Health and Medical Research Council; NYHA: New York Heart Association; PDAs: Personal digital assistants; QOL: Quality of Life; RCT: Randomised Control Trial; SBP: Systolic Blood Pressure; SCG: Standard care alone group; SMS: Short messaging service; TMG: Telemonitoring group}

\section{Acknowledgements}

Dr. Hamilton is a Research Fellow and acknowledges support from the UWA Poche Centre of Indigenous Health. The WA Centre for Rural Health receives core funding from the Australian Department of Health.

\section{Funding}

Not applicable

Availability of data and materials

Studies included in this systematic review are available online.

\section{Authors' contributions}

$\mathrm{SH}$ contributed to the systematic review's conception and research question, data base search, analysis, interpretation and writing. BM contributed to systematic review's conception and research question, data base search, analysis and writing. EMB contributed to the data base search, analysis and writing. SCT contributed to the systematic review's conception and research question, analysis, interpretation and writing. All authors approved the final manuscript.

Ethics approval and consent to participate

Not applicable

Consent for publication

Not applicable

\section{Competing interests}

The authors declare that they have no competing interests.

\section{Publisher's Note}

Springer Nature remains neutral with regard to jurisdictional claims in published maps and institutional affiliations.

\section{Author details}

${ }^{1}$ Western Australian Centre for Rural Health, University of Western Australia, 35 Stirling Highway, Crawley, WA 6009, Australia. ${ }^{2}$ Georgetown University, 3700 O St NW, Washington, DC 20057, USA.

Received: 25 March 2017 Accepted: 31 January 2018

Published online: 07 February 2018

\section{References}

1. National Health Priority Action Council (NHPAC). National Chronic Disease Strategy. Canberra: Australian Government Department of Health and Aging; 2006.

2. Australian Institute of Health and Welfare. Health-care expenditure on cardiovascular diseases 2008-09. Cat. no. CVD 65. Canberra: AlHW; 2014. 
3. National Heart Foundation of Australia \& Australian Cardiac Rehabilitation Association. Recommended Framework for Cardiac Rehabilitation 04. Melbourne: National Heart Foundation of Australia; 2004.

4. Briffa TG, Kinsman L, Maiorana AJ, Zecchin R, Redfern J, Davidson PM, Paull G, Nagle A, Denniss AR. An integrated and coordinated approach to preventing recurrent coronary heart disease events in Australia. Med J Aust. 2009;190(12):683-6.

5. Clark RA, Conway A, Poulsen V, Keech W, Tirimacco R, Tideman P. Alternative models of cardiac rehabilitation: a systematic review. Eur J Prev Cardiol. 2015;22(1):35-74.

6. National Heart Foundation of Australia \& Australian Cardiac Rehabilitation Association. Recommended Framework for Cardiac Rehabilitation 04. Melbourne: National Heart Foundation of Australia; 2004. www. heartfoundation.org.au.

7. National Heart Foundation of Australia: Secondary prevention of cardiovascular disease. In.; 2010

8. Briffa T, Kinsman L, Maiorana A, Zecchin R, Redfern J, Davidson P, Paull G, Nagle A, Denniss A. An integrated and coordinated approach to preventing recurrent coronary heart disease events in Australia. Policy statement from the Australian cardiovascular health and rehabilitation association. Med J Aust. 2009;190(12):683-6.

9. Shepherd F, Battye K, Chalmers E. Improving access to cardiac rehabilitation for remote indigenous clients. Aust NZ J Public Health. 2003;27(6):632-6.

10. Redfern J, Maiorana A, Neubeck L, Clark AM, Briffa T. Achieving coordinated secondary prevention of coronary heart disease for all in need (SPAN). Int J Cardiol. 2011;146(1):1-3.

11. Redfern J, Ellis E, Briffa T, Freedman S. High risk-factor level and low riskfactor knowledge in patients not accessing cardiac rehabilitation after acute coronary syndrome. Med J Aust. 2007;186(1):21-5.

12. Australian Bureau of Statistics; Australian Social Trends March 2011: Health outside major cities. http://www.abs.gov.au/ausstats/abs@.nsf/ Previousproducts/4102 OMain\%20Features30Mar\%202011?opendocument\&tabname= Summary\&prodno=4102.0\&issue=Mar\%202011\&num =\&view $=$

13. Australian Bureau of Statistics; 2011 Census Community Profiles. http:// www.censusdata.abs.gov.au/census_services/getproduct/census/2011/ communityprofile/0.

14. Levesque JF, Harris MF, Russell G. Patient-centred access to health care: conceptualising access at the interface of health systems and populations. Int J Equity Health. 2013;12:18.

15. DiGiacomo M, Davidson PM, Taylor KP, Smith JS, Dimer L, Ali M, Wood MM, Leahy TG, Thompson SC. Health information system linkage and coordination are critical for increasing access to secondary prevention in aboriginal health: a qualitative study. Qual Prim Care. 2010;18(1):17-26.

16. Higgins RO, Murphy BM, Grande MRL, Parkinson A, Worcester MUC, Goble AJ. Expressed preferences for health education of patients after percutaneous coronary intervention. Euro J Cardiov Prev R. 2005; 12(6):572-9.

17. Widmer RJ, Collins NM, Collins CS, West CP, Lerman LO, Lerman A. Digital health interventions for the prevention of cardiovascular disease: a systematic review and meta-analysis. Mayo Clin Proc. 2015;90(4):469-80.

18. Park LG, Beatty A, Stafford Z, Whooley MA. Mobile phone interventions for the secondary prevention of cardiovascular disease. Prog Cardiovasc Dis. 2016;58(6):639-50.

19. García-Lizana F, Sarría-Santamera A. New technologies for chronic disease management and control: a systematic review. J Telemed Telecare. 2007; 13(2):62-8.

20. Varnfield M, Karunanithi M. Information and communication technologybased cardiac rehabilitation homecare programs. Smart Homecare Technol TeleHealth. 2015;3:69-79.

21. Australian Communications and Media Authority: Communications report; 20132014. http://www.acma.gov.au/ /media/Research\%20and\%20Analysis/Publication/ Comms\%20Report\%202013\%2014/PDF/Communications\%20report\%20201314_ LOW-RES\%20FOR\%20WEB\%20pdf.pdf.

22. Mackay M. Australian mobile phone lifestyle index. 10th ed. Australia: AIMIA 2014. http://www.aimia.com.au/ampli2014

23. Nilsen W, Kumar S, Shar A, Varoquiers C, Wiley T, Riley W, Pavel M, Atienza A. Advancing the science of mHealth. J Health Commun. 2012:17:5-10.

24. World Health Organisation: mHealth: new horizons for health through mobile technologies: second global survey on eHealth. In.; 2011, http:// apps.who.int/iris/bitstream/10665/44607/1/9789241564250_eng.pdf.
25. Stephens J, Allen J. Mobile phone interventions to increase physical activity and reduce weight: a systematic review. J Cardiovasc Nurs. 2013;28(4):320-9.

26. Kumar S, Nilsen WJ, Abernethy A, Atienza A, Patrick K, Pavel M, Riley WT, Shar A, Spring B, Spruijt-Metz D, et al. Mobile health technology evaluation: the mHealth evidence workshop. Am J Prev Med. 2013;45(2):228-36.

27. Joanna Briggs Institute. Joanna Briggs Institute Reviewers' Manual. 2011th ed: The Joanna Briggs Institute; 2011.

28. National Health and Medical Research Council. How to use the evidence: assessment and application of scientific evidence: Handbook series on preparing clinical practice guidelines: Commonwealth of Australia; 2000. http://joannabriggs.org/assets/docs/sumari/reviewersmanual-2011.pdf

29. Critical Appraisal Skills Programme (CASP). Oxford: CASP; 2014. http:// joannabriggs.org/assets/docs/sumari/reviewersmanual-2011.pdf.

30. Worringham C, Rojek A, Stewart I, Miranda J. Development and feasibility of a smartphone. ECG and GPS based system for remotely monitoring exercise in cardiac rehabilitation. PLoS One. 2011;6:e14669.

31. Varnfield M, Karunanithi MK, Sarela A, Garcia E, Fairfull A, Oldenburg BF, Walters DL. Uptake of a technology-assisted home-care cardiac rehabilitation program. Med J Aust. 2011;194(4):S15-9.

32. Varnfield M, Karunanithi M, Lee CK, Honeyman E, Arnold D, Ding H, Smith C, Walters DL. Smartphone-based home care model improved use of cardiac rehabilitation in postmyocardial infarction patients: results from a randomised controlled trial. Heart. 2014;100(22):1770-9.

33. Whittaker R, Merry S, Dorey E, Maddison R. A development and evaluation process for mHealth interventions: examples from New Zealand. J Health Commun. 2012;17:11-21.

34. Pfaeffli L, Maddison R, Whittaker R, Stewart R, Kerr A, Jiang Y, Kira G, Carter K, Dalleck L. A mHealth cardiac rehabilitation exercise intervention: findings from content development studies. BMC Cardiovasc Disord. 2012;12:36.

35. Forman D, LaFond K, Panch T, Allsup K, Manning K, Sattelmair J. Utility and efficacy of a smartphone application to enhance the learning and behavior goals of traditional cardiac rehabilitation: a feasibility study. J Cardiopulm Rehabil Prev. 2014;34(5):327-34.

36. Blasco A, Carmona M, Fernandez-Lozano I, Salvador CH, Pascual M, Sagredo PG, Somolinos R, Munoz A, Garcia-Lopez F, Escudier JM, et al. Evaluation of a telemedicine service for the secondary prevention of coronary artery disease. J Cardiopulm Rehabil Prev. 2012;32(1):25-31.

37. Scherr D, Zweiker R, Kollmann A, Kastner P, et al. Mobile phone-based surveillance of cardiac patients at home. J Telemed Telecare. 2006;12(5):255-61.

38. Scherr D, Kastner P, Kollmann A, Hallas A, Auer J, Krappinger H, Schuchlenz H, Stark G, Grander W, Jakl G, et al. Effect of home-based Telemonitoring using mobile phone technology on the outcome of heart failure patients after an episode of acute decompensation: randomized controlled trial. J Med Internet Res. 2009;11(3):e34

39. Seto E, Leonard KJ, Cafazzo JA, Barnsley J, Masino C, Ross HJ. Mobile phonebased Telemonitoring for heart failure management: a randomized controlled trial. J Med Internet Res. 2012;14(1):e31.

40. Vuorinen AL, Leppanen J, Kaijanranta H, Kulju M, Helio T, van Gils M, Lahteenmaki J. Use of home telemonitoring to support multidisciplinary care of heart failure patients in Finland: randomized controlled trial. J Med Internet Res. 2014:16(12):e282.

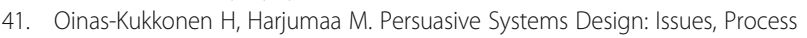
Model,and System Features. Communications of the AIS. 2009;24(28).

42. Webb TL, Joseph J, Yardley L, Michie S. Using the internet to promote health behavior change: a systematic review and meta-analysis of the impact of theoretical basis, use of behavior change techniques, and mode of delivery on efficacy. J Med Internet Res. 2010;12(1):e4.

43. Fogg B. A behavior model for persuasive design. In: Proceedings of the 4th international conference on persuasive technology. Claremont, California: ACM; 2009. p. 1-7.

44. Kelders MS, Kok NR, Ossebaard CH, Van Gemert-Pijnen EWCJ. Persuasive system design does matter: a systematic review of adherence to web-based interventions. J Med Internet Res. 2012;14(6):e152.

45. Beatty AL, Fukuoka Y, Whooley MA. Using mobile Technology for Cardiac Rehabilitation: a review and framework for development and evaluation. JAMA. 2013;2(6)

46. Riley WT, Rivera DE, Atienza AA, Nilsen W, Allison SM, Mermelstein R. Health behavior models in the age of mobile interventions: are our theories up to the task? Transl Behav Med. 2011;1(1):53-71. 
47. Dale LP, Whittaker R, Eyles H, Mhurchu CN, Ball K, Smith N, Maddison R. Cardiovascular disease self-management: pilot testing of an mHealth healthy eating program. J Pers Med. 2014;4(1):88-101.

48. Craig P, Dieppe P, Macintyre S, Michie S, Nazareth I, Petticrew M. Developing and evaluating complex interventions: the new Medical Research Council guidance. Br Med J. 2008;337:a1655.

49. Tomlinson M, Rotheram-Borus MJ, Swartz L, Tsai AC. Scaling up mHealth: where is the evidence? PLoS Med. 2013;10(2):e1001382.

50. Glasgow RE, Linnan LA: Evaluation of Theory-based Interventions. In: Health Behavior and Health Education: Theory, Research and Practice. Fourth edn. Edited by Glanz K RB, Viswanath K. Wiley, Hoboken; 2008: 487-508.

51. Hallberg I. Knowledge for Health Care Practice. In: HI RDA, editor. Complex Interventions in Health: An overview of research methods. Oxon: Routledge: 2015. p. 16-28.

52. National Heart Foundation of Australia. My heart, my life: A guide for people living with coronary heart disease, 2nd edition. https://www. heartfoundation.org.au/images/uploads/publications/CON-141.v4_ MHML2015_WEB.PDF.

53. Antypas K, Wangberg SC. An internet- and mobile-based tailored intervention to enhance maintenance of physical activity after cardiac rehabilitation: short-term results of a randomized controlled trial. J Med Internet Res. 2014;16(3):e77.

54. Fletcher SM, Burley MB, Thomas KE, Mitchell EK. Feeling supported and abandoned: mixed messages from attendance at a rural community cardiac rehabilitation program in Australia. J Cardiopulm Rehabil Prev. 2014;34(1): 29-33.

55. Lancaster G, Campbell M, Eldridge S, Farrin A, Marchant M, Muller S. Trials in primary care: statistical issues in the design, conduct and evaluation of complex interventions. Stat Methods Med Res. 2010;19:349-77.

56. Craig P, Dieppe P, Macintyre S, Michie S, Nazareth I, M P: Developing and evaluating complex interventions: new guidence. https://www.mrc.ac.uk/ documents/pdf/complex-interventions-guidance/. In.

57. Frederix I, Hansen D, Coninx K, Vandervoort P, Vandijck D, Hens N, Van Craenenbroeck E, Van Driessche N, Dendale P. Medium-term effectiveness of a comprehensive internet-based and patient-specific Telerehabilitation program with text messaging support for cardiac patients: randomized controlled trial. J Med Internet Res. 2015:17(7):e185.

58. Maddison R, Pfaeffli L, Whittaker R, Stewart R, Kerr A, Jiang Y, Kira G, Leung W, Dalleck $L$, Carter K, et al. A mobile phone intervention increases physical activity in people with cardiovascular disease: results from the HEART randomized controlled trial. Eur J Prev Cardiol. 2015;22(6):701-9.

59. Woodruffe S, Neubeck L, Clark RA, Gray K, Ferry C, Finan J, Sanderson S, Briffa TG. Australian cardiovascular health and rehabilitation association (ACRA) Core components of cardiovascular disease secondary prevention and cardiac rehabilitation 2014. Heart Lung Circ. 2015;24(5):430-41.

\section{Submit your next manuscript to BioMed Central and we will help you at every step:}

- We accept pre-submission inquiries

- Our selector tool helps you to find the most relevant journal

- We provide round the clock customer support

- Convenient online submission

- Thorough peer review

- Inclusion in PubMed and all major indexing services

- Maximum visibility for your research

Submit your manuscript at www.biomedcentral.com/submit

) Biomed Central 